\title{
Interactions of large-scale free-stream turbulence with turbulent boundary layers
}

\author{
Eda $\operatorname{Dogan}^{1} \dagger$, Ronald Hanson ${ }^{1}$ and Bharathram \\ Ganapathisubramani ${ }^{1}$ \\ ${ }^{1}$ Aerodynamics and Flight Mechanics Research Group, University of Southampton, \\ Southampton SO17 1BJ, UK
}

(Received $\mathrm{xx}$; revised $\mathrm{xx}$; accepted $\mathrm{xx}$ )

The scale interactions occurring within a turbulent boundary layer are investigated in the presence of free-stream turbulence. The free-stream turbulence is generated by an active grid. The free stream is monitored by a single-component hot-wire probe while a second probe is roved across the height of the boundary layer at the same streamwise location. Large-scale structures occurring in the free-stream are shown to penetrate the boundary layer and increase the streamwise velocity fluctuations throughout. It is speculated that depending on the extent of the penetration, i.e. based on the level of freestream turbulence, the near-wall turbulence production peaks at different wall-normal locations than the expected location of $y^{+} \approx 15$ for a canonical turbulent boundary layer. It is shown that the large-scales dominating the log region have a modulating effect on the small-scales in the near wall region; this effect becomes more significant with increasing turbulence in the free-stream, i.e. similarly increasing $R e_{\lambda 0}$. This modulating interaction and its Reynolds number trend have similarities with canonical turbulent boundary layers at high Reynolds numbers where the interaction between the large-scales and the envelope of the small-scales exhibit a pure amplitude modulation (Hutchins \& Marusic $2007 a$; Mathis et al. 2009). This similarity has encouraging implications toward generalising scale interactions in turbulent boundary layers.

\section{Introduction}

Coherent structures within the turbulent boundary layer are known to be dominated by energetic large-scales at high Reynolds numbers. They are populated in the outer region of the boundary layer and carry a significant portion of the turbulent kinetic energy, which contributes to almost half of the Reynolds shear stress in the boundary layer (Guala et al. 2006; Balakumar \& Adrian 2007). These large-scale structures are also found to exhibit a footprint in the near-wall region affecting the near-wall structures (Hutchins \& Marusic 2007a). This has significant relevance in an engineering context since dragreduction control strategies are usually based on manipulating the near-wall structures (Smits et al. 2011) and factors influencing these structures will have implications toward effective control strategies.

Bandyopadhyay \& Hussain (1984) examined the interaction between the large and small scales in various shear flows, including boundary layers, mixing layers, wakes and jets. They found significant coupling between these scales across all shear flows by correlating the low-frequency component (i.e. low-pass filtered time series data from hot-wire measurements) with a signal similar to the envelope of the high-frequency component. Later, Hutchins \& Marusic (2007a) showed that the large-scales tend to modulate the

$\dagger$ Email address for correspondence: E.Dogan@soton.ac.uk 
amplitude of the small-scale fluctuations. Mathis et al. (2009) expanded upon this nonlinear interaction and defined the modulation by a correlation coefficient between the large-scale streamwise fluctuating velocity and an envelope of the small-scale fluctuations to quantify the level of modulation across the boundary layer. Ganapathisubramani et al. (2012), on the other hand, explored the dependence of the level of modulation on the strength of the large-scale fluctuations. In addition, they showed significant frequency modulation of the small-scale fluctuations by the large-scales. Drawing from these studies, a predictive model for the near-wall turbulence was proposed with only large-scale information input (Mathis et al. 2011a; Marusic et al. 2010).

The previous studies of Hutchins \& Marusic (2007a) and Mathis et al. $(2009,2011 a)$ consider canonical turbulent boundary layers. Whereas Jacobi \& McKeon $(2011 a, b, 2013)$ and Duvvuri \& McKeon (2015) introduced a synthetic large-scale into the boundary layer to generalise the influence of large-scale structures on the small-scales. Jacobi \& McKeon (2013) showed that very large scale motions $(6 \delta-10 \delta)$ are the primary modulating scale. Furthermore, Duvvuri \& McKeon (2015) indicated that exciting the turbulent boundary layer by a large-scale input modifies the phase relationships naturally existing between the scales. These relationships are quantified by skewness and the amplitude modulation coefficient as previously suggested by Chung \& McKeon (2010) and later confirmed by Ganapathisubramani et al. (2012). While the studies of Jacobi \& McKeon (2013) and Duvvuri \& McKeon (2015) introduce a single large-scale into the boundary layer, we input a wide range of large-scales that are introduced from outside the boundary layer in the free-stream. In this paper, we explore the effects of an external disturbance, namely free-stream turbulence, on the turbulent boundary layer. Specifically the focus is given toward the influence on the scale interactions.

Free-stream turbulence (FST) exists above almost all naturally and industrially occurring turbulent boundary layers (Sharp et al. 2009); therefore, in an engineering context, it is important to investigate how the turbulent boundary layer responds to FST. For the turbulent boundary layer, increased FST is widely known to cause increased skin friction and enhanced heat transfer (Hancock \& Bradshaw 1983; Blair 1983; Castro 1984). The focus of these studies was to find parameters to correlate the increases of skin friction and heat transfer with FST. Hancock \& Bradshaw (1983) concluded that the effect of FST was not only dependent on the turbulence intensity level but also on a characteristic length scale of FST which they defined as dissipation length scale. They formed a FST parameter using both the turbulence intensity and length scale to define the overall effect of the FST on turbulent boundary layers. Blair (1983) added a damping term to this parameter to account for low Reynolds number effects. Later, Thole \& Bogard (1996) presented boundary layer statistics for FST levels up to $20 \%$ and confirmed the validity of the log-law in the mean profiles of the boundary layer for these high turbulence levels by direct measurements of total shear stress. Stefes \& Fernholz (2004) complemented the study of Thole \& Bogard (1996) by providing skin friction data and measurements in the vicinity of the wall at relatively higher Reynolds numbers at FST levels up to $13 \%$. They also presented mean and fluctuating velocity distributions for the boundary layer and showed that there were considerable changes in the outer region of the boundary layer such as the increase in the velocity fluctuations.

FST adds multiple length scales into the boundary layer and changes the nature of momentum and energy transport through scale interactions. Sharp et al. (2009) examined these scale interactions. They suggested that depending on its level, FST can penetrate into the boundary layer as well as affecting the small near-wall scales. However, the nearest wall-normal measurement location presented in their study is around $y^{+} \approx 50$ where $y^{+}=y U_{\tau} / \nu$ and $y$ is the wall-normal distance, $U_{\tau}$ is the skin-friction velocity and $\nu$ is 
the kinematic viscosity. Therefore, the near-wall information is needed to establish a clear relationship between the near-wall turbulence and FST level and also to determine the extent of the FST penetration into the near-wall region. Currently the mechanism on how FST interacts with the outer region of the boundary layer and how this in turn affects the near-wall turbulence, specifically in the context of scale interactions, is not well understood. With these motivations in hand, this paper aims to contribute towards the understanding of the scale interactions in a turbulent boundary layer in the presence of FST. Here we provide measurements of a turbulent boundary layer using hot-wire anemometry. The measurements could reach the wall down to $y^{+} \approx 5$ which have the potential to reveal the near-wall information needed in the literature for a turbulent boundary layer under the effect of FST. Free-stream is also characterised in detail by hot-wire measurements and different FST conditions of increasing turbulence levels are investigated in detail in the paper.

\section{Experiments}

\subsection{Facility}

The experiments were performed in a suction type wind tunnel at the University of Southampton. The test section of the tunnel is $4.5 \mathrm{~m}$ long and has a cross-section of 0.9 x $0.6 \mathrm{~m}^{2}$. A turbulent boundary layer was established on a $4.2 \mathrm{~m}$ long flat-plate mounted $0.135 \mathrm{~m}$ above the floor of the test section. The plate had a sharp leading edge (machined from aluminium) that was followed by a COMPOCEL $\mathrm{R}$ sandwich panel of parallel 1 $\mathrm{mm}$ thick aluminium sheets bonded to a $8 \mathrm{~mm}$ thick aluminium honeycomb core. A 0.5 $\mathrm{m}$ long flap followed at the trailing edge of the plate. The flap was used to position the stagnation point on the measurement side of the plate. The leading edge of the plate was positioned $0.3 \mathrm{~m}$ downstream of the active grid. The active grid was placed between the contraction and the test-section of the tunnel. Although the grid-generated FST could trip the boundary layer to a turbulent state, the leading edge was also equipped with a tripping wire that was necessary for measurements made at low FST levels.

The design of the active grid used to generate the FST was based on that of Makita (1991) and similar later designs such as those found in Mydlarski \& Warhaft (1996); Kang et al. (2003); Larssen \& Devenport (2011); Hearst \& Lavoie (2015). The active grid has a square mesh of length $(M)$ of $81 \mathrm{~mm}$. To account for the rectangular test section, the active grid has $11 \times 7$ rods, each with a diameter of $10 \mathrm{~mm}$. Square wings of length $55.86 \mathrm{~mm}$ are attached to the rods as shown in figure 1b. Two sets of square wings have been used: one solid set and the other of the same outer dimension but with reduced solidity using circular cutouts (see figure $1 \mathrm{a}$ and $1 \mathrm{~b}$, respectively). The maximum frontal blockage by the solid wings is $100 \%$ and this has been reduced to $75 \%$ for the circular cut-out wings. Each of the rods could be rotated independently using stepper motors. The motors were daisy-chained and controlled through a MATLAB routine. By using a combination of different rotational schemes, free-stream velocities, $U_{0}$, and with the two different sets of wings, a wide range of FST cases was possible. The turbulence intensity levels considered ranged from around $7 \%$ to around $13 \%$.

\subsection{Experimental Methods}

Measurements of the streamwise flow velocity in the boundary layer and in the freestream were made using two single-component hot-wire anemometers. Each hot-wire probe was located at the mid-span of the boundary layer plate and $43 M$ downstream of the active grid. One probe was held stationary at $27 \mathrm{~cm}$ above the flat plate in 


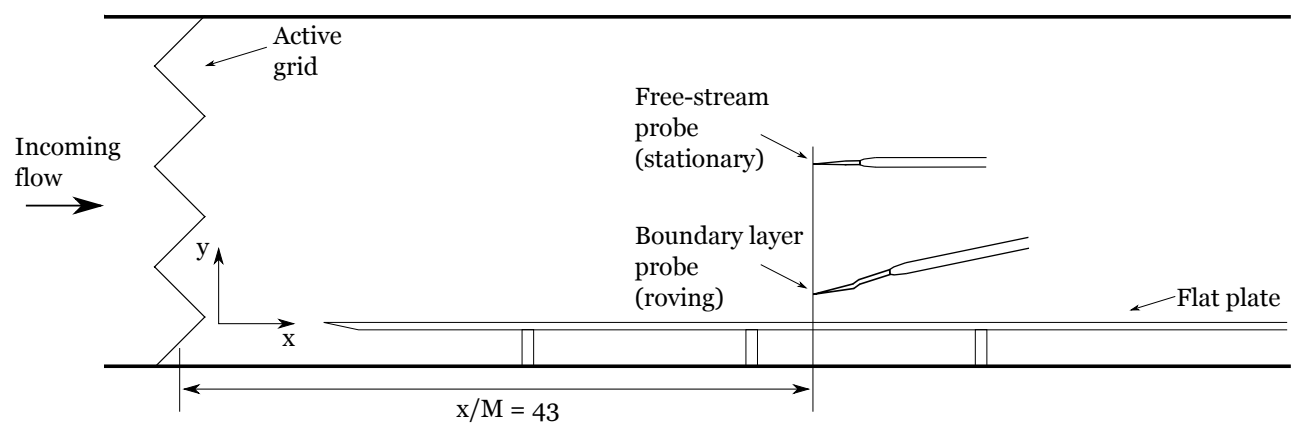

(a)
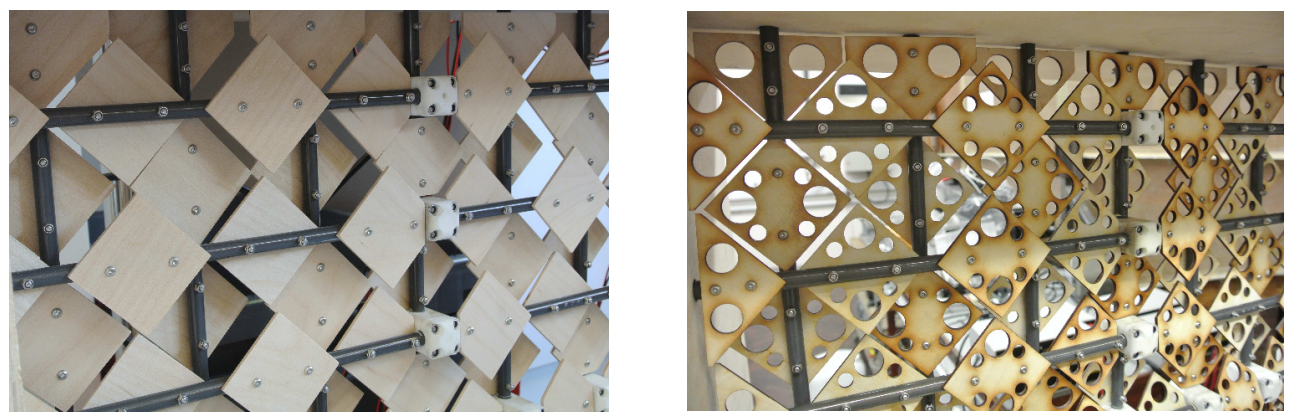

(b)

Figure 1: (a) Schematic of the test section (b) different sets of wings used during the experiment: solid wings (left), cut-out wings (right).

the free-stream, whereas the other could be traversed from the wall, $y=0$, to the free stream as shown in figure 1a. The single wire traversing the boundary layer was an Auspex A55P05 boundary layer probe that had $10 \mathrm{~mm}$ long prongs spanned by a $3 \mathrm{~mm}$ long and $5 \mu \mathrm{m}$ diameter tungsten wire with a central $1.05 \mathrm{~mm}$ active region shouldered on either side by copper-plated sections. The single wire located in the free stream was an Auspex A55P01 probe with the same active sensor length as the boundary layer probe. The resulting length to diameter ratio of each probe was 210 following the recommendation by Ligrani \& Bradshaw (1987) and Hutchins et al. (2009). A Dantec StreamLine Pro Constant Temperature Anemometer (CTA) system was used to operate the hot-wire probes with an overheat ratio set to 1.8. The output of the CTA system was low-pass filtered at $10 \mathrm{kHz}$ and was sampled at a rate of $20 \mathrm{kHz}$, to prevent aliasing, by a 16-bit National Instruments USB-6212 BNC, A/D data acquisition board, connected to a PC. Measurements of the streamwise velocity in the boundary layer were acquired at 28 wall-normal locations for a sampling period of 6 minutes. The streamwise velocity in the free-stream was acquired simultaneously. The hot-wires were statically calibrated using a 14-point velocity calibration ranging between $1.4 \mathrm{~m} / \mathrm{s}$ and $14 \mathrm{~m} / \mathrm{s}$. The reference velocity was determined using a pitot-static tube connected to a Furness FCO510 0 - 200 Pa pressure transducer. The temperature of the free-stream was acquired by a T-type Omega thermocouple throughout the experiments. Although the ambient temperature variation remained within \pm 0.9 degrees Celcius, hot-wire output signals were corrected with respect to the reference temperature of the calibration (see Bruun (1995)). Regardless, calibrations were performed immediately before (pre-) and after 
(post-) each experiment to linearly correct for drift between pre- and post-calibrations. The calibrations were performed while the wings of the grid were aligned with the freestream direction, i.e. similar to a static mesh grid having turbulence levels of around $2-2.5 \%$. This calibration method was validated against a test with the grid removed and agreed within $\pm 1 \%$. Therefore, calibrations in all experiments reported were performed without removing the active grid.

The friction velocity was measured using the Preston tube method. For this method, the pressure difference was measured between two tubes, one that is placed in contact with the wall and open to the oncoming flow, whereas the other is a static pressure port drilled through the surface of the plate at the same streamwise location. This pressure difference was measured using Furness FCO510 0 - 200 Pa pressure transducer. The total pressure tube was given a minor curvature and fixed with tape at the centre of its 15 $\mathrm{cm}$ length to ensure that the tube opening was in contact with the plate at all times. The tube placed in contact with the wall had a diameter of $2.76 \mathrm{~mm}$ and the static port diameter was $1 \mathrm{~mm}$. This method is dependent on the assumption of the log-law validity (Patel 1965), which will be shown to be valid for the boundary layers considered in this study. This method also required a calibration relating friction velocities derived from log-law to the difference between the total pressure measured by Preston tube and the static pressure measured from the static port. For this calibration, $U_{\tau}$ was measured using the Clauser chart method for the no-FST case over a range of $R e_{\tau}$ that corresponded to the range of measured FST cases during the course of this study. Then, an in-house calibration was devised to relate these $U_{\tau}$ values to the pressure differences measured simultaneously using Preston tubes. We have found excellent agreement (within the experimental uncertainty) with our own calibration and the calibration by Patel (1965) for Preston tube measurements. The results from Preston tube were also validated by oil film interferometry measurements which were carried out later under the same conditions.

\section{Results}

\subsection{Free-stream conditions}

The FST cases were generated using the active grid by rotating the shafts at specified rotation rates during specified periods. Two different sets of wings, i.e. solid wings and cut-out wings, were used as described previously to achieve a range of turbulence intensity values from approximately $7 \%$ to $13 \%$. The motor schemes of the active grid were reproduced from various active grid studies in the literature (Kang et al. 2003; Larssen \& Devenport 2011). A total of 20 FST cases were documented during the course of this study (see table 1 and table 3 ). Detailed analysis is generally limited to only the four cases given in table 1 for the sake of brevity and because the phenomena of interest to this study can be adequately described from this reduced set. However, we include trends determined from the inclusive set of 20 cases when broader points are made. For the cases investigated in detail (given in table 1) the operation parameters of the active grid were reproduced from Larssen \& Devenport (2011) (test case 14 in their study). It involves setting a uniformly distributed rotational speed in the range of 2-6 Hz for each rod's motor once every second while adjacent motors rotate in the opposite direction.

The characteristics of the four FST cases investigated in this paper are summarised in table 1 . Using the solid or reduced blockage sets of wings mainly resulted in different levels of turbulence intensity. Cases A and B were generated using cut-out wings resulting in what we will refer to as "low-range" turbulence intensity levels around $7-8 \%$. Cases $\mathrm{C}$ and $\mathrm{D}$ were generated using solid wings resulting in what we will refer similarly to as 


$\begin{array}{ccccccccc}\text { FST cases } & & U_{0}(m / s) & \sqrt{\overline{u_{0}^{2}}} / U_{0}(\%) & R e_{\lambda 0} & L_{0} / M & L_{0}^{*} / M & \lambda_{0}(m m) & \eta_{0}(m m) \\ \text { A } & (\triangle) & 6.1 & 7.4 & 315 & 2.1 & 5.6 & 10.8 & 0.3 \\ \text { B } & (\diamond) & 10.1 & 8.3 & 505 & 2.8 & 4.2 & 9.4 & 0.2 \\ \text { C } & (\triangleright) & 8.0 & 12.1 & 530 & 2.7 & 3.4 & 8.4 & 0.2 \\ \text { D } & (\mathbb{\square}) & 9.9 & 12.7 & 645 & 3.0 & 3.0 & 7.9 & 0.2\end{array}$

Table 1: (Colour online) Free-stream parameters for the study cases at $x=43 M$. $U_{0}$ : mean streamwise velocity, $\sqrt{\overline{\overline{u_{0}^{2}}}} / U_{0}(\%)$ : turbulence intensity, $R e_{\lambda 0}$ : Reynolds number based on the Taylor micro-scale, $L_{0} / M$ and $L_{0}^{*} / M$ : integral length scale ratio based on $M, \lambda_{0}$ : Taylor micro-scale, $\eta_{0}$ : Kolmogorov scale. Detailed definitions of the parameters are given in the text.

"high-range" turbulence intensity levels around $12-13 \%$. The parameters are obtained from the measurements acquired at $43 M$ downstream of the grid. The subscript 0 is used to denote the free-stream values throughout the paper. Quantities with this subscript were obtained from the stationary probe in the free-stream. The instantaneous velocity in the streamwise direction is denoted by the sum of the mean and fluctuating components, $U+u$. Reynolds number, $R e_{\lambda_{0}}$, based on the Taylor micro-scale, $\lambda_{0}$, is defined as $\sqrt{\overline{u_{0}^{2}}} \lambda_{0} / \nu$ where $\lambda_{0}$ is calculated as $\sqrt{\left(15 \nu \overline{u_{0}^{2}} / \epsilon\right)}$. Within this definition, the turbulence energy dissipation rate, $\epsilon$, is calculated from the spatial gradient of the streamwise velocity fluctuations as $15 \nu \overline{\left(\partial u_{0} / \partial x\right)^{2}}$, the formulation of which invokes Taylor's hypothesis to obtain the spatial gradient from the temporal gradient calculated from the hot-wire measurements. To improve the estimate of the gradient quantities in a turbulent flow from hot-wire measurements, higher order differencing schemes are suggested (Hearst et al. 2012); therefore 7-point centred differencing schemes were employed in this study to calculate the velocity gradients. Using the dissipation rate, $\epsilon$, Kolmogorov scale $\left(\eta_{0}\right)$, can be calculated from $\left(\nu^{3} / \epsilon\right)^{1 / 4}$. There are several ways to define the integral length scale $\left(L_{0}\right)$ in a turbulent flow. A common definition is to use the value of the integrated normalised autocorrelation to the first zero crossing. However, it was found that the low frequency energy content caused by the active grid prevented the autocorrelation from reaching zero. For this reason a value of 0.1 was chosen in the present study to determine the extent of the integration region, which was above the asymptotic limit reached in all cases. Another common method to calculate the integral length scale often used in active grid studies is from the wavenumber at which the pre-multiplied spectra peaks. Since this method is associated with significant scatter, Mydlarski \& Warhaft (1996) introduced the relation, $L_{0}^{*}=0.9 \overline{u_{0}^{2}} / \epsilon$. The integral length scale using each of the aforementioned methods is included in table 1 for comparison purposes. As will become clear later, this study is particularly interested in the most energetic free-stream scales, which differ from the tabulated integral length scales.

The decay of turbulence downstream of a grid is governed by a power law as $\overline{u_{0}^{2}} / U_{0}^{2}=$ $A(x / M)^{n}$ as given in Mydlarski \& Warhaft (1996). For each of the four cases described in table 1 , figure 2 shows the decay of the variance of streamwise velocity fluctuations, $\overline{u_{0}^{2}}$. The measurements were performed along the test section midway between the test- 


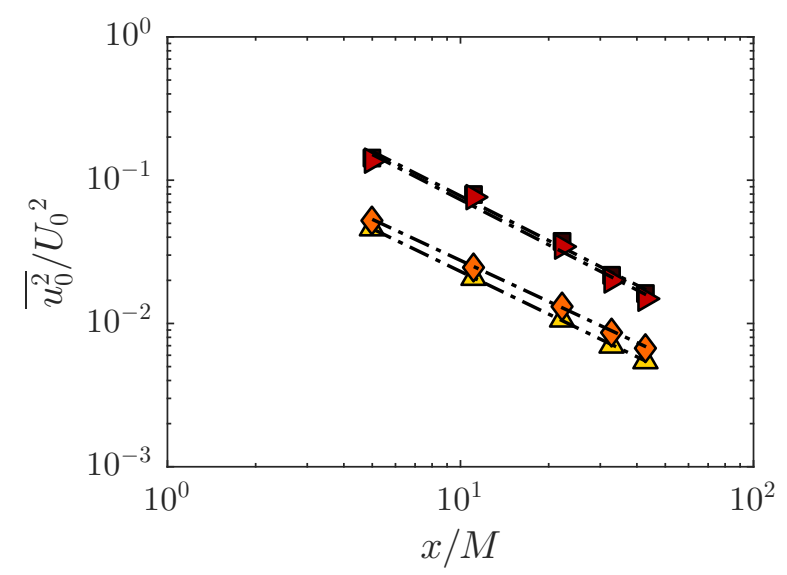

Figure 2: (Colour online) Decay of the normalised variance of the streamwise velocity fluctuations for the FST cases. $(\triangle)$ : case A, $(\diamond)$ : case B, $(\triangleright)$ : case C and $(\square)$ : case D.

section ceiling and the measurement side of the plate from approximately $x / M=5$ until the fixed downstream location for the boundary layer profiles, $x / M=43$. For these cases, an exponent of $n=1.25$ adequately describes the observed decay shown in this figure. This value is consistent with similar active grid studies from the literature (Mydlarski \& Warhaft 1996; Kang et al. 2003). The vertical shift in figure 2 between two different sets of lines, i.e. different constants of $A$ in the power-law equation, is caused by the solidity, i.e. the open area ratio, of the grid. Regardless, it can be deduced that the decay of turbulence is similar for each of the different FST cases. This suggests that the similarities and/or differences between different boundary layer cases presented in this study is not related to the rate of turbulence decay.

At the downstream location of the boundary layer profiles, $43 M$, figure 3 shows the pre-multiplied energy spectra of the streamwise velocity fluctuations in the free-stream normalised by the local free-stream variance, $k_{x} \phi_{u u} / \overline{u_{0}^{2}}$, with the streamwise wavelength, $\zeta_{x}$, normalised by the grid mesh length, $M$, for the FST cases. The non-dimensional spectra are similar for each case and exhibit significant collapse. The characteristic wavelength of the spectral peak occurs at approximately $20 M$, which is several times longer than the integral length scales given in table 1. Given that the length scale of approximately $20 M$ represents the most energetic scale, it is also chosen as the representative scale of turbulence in the free-stream.

\subsection{Boundary layer characteristics}

A zero-pressure gradient turbulent boundary layer was established under the influence of FST on a smooth flat plate as described previously in $\S 2.1$. Herein, the characteristics of the turbulent boundary layers developed under the influence of different FST conditions, primarily those described in table 1 and with inclusive dataset described in table 3 (where appropriate or relevant) will be discussed for the rest of the paper. In addition, in the instances where comparison is possible they will be presented together with the baseline case taken without the presence of the active grid, which will be referred to as "no-FST".

Table 2 is a summary of the turbulent boundary layer parameters for the no-FST case and for the four FST cases chosen for this paper. This table is an extension of table 1 for the parameters of the boundary layers developed under the effect of the corresponding 


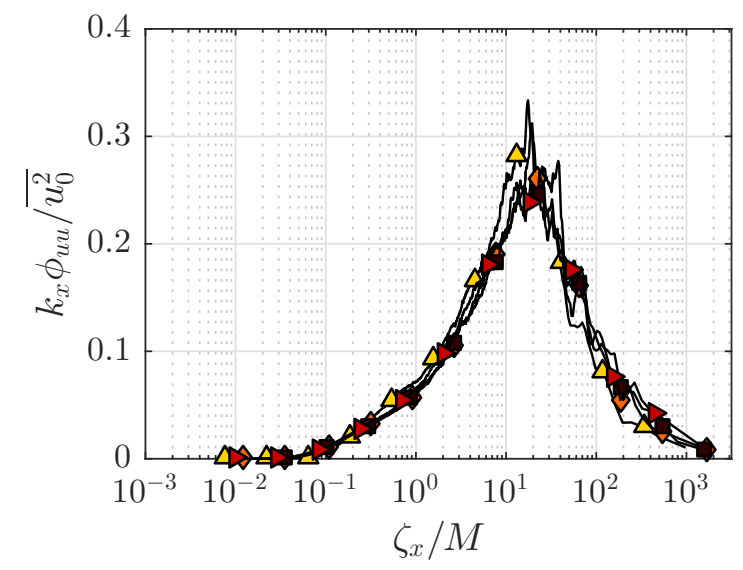

Figure 3: (Colour online) Normalised pre-multiplied energy spectra of streamwise velocity fluctuations, $k_{x} \phi_{u u} \overline{u_{0}^{2}}$, of the free-stream for the FST cases with streamwise wavelength, $\zeta_{x}$, of the free-stream with respect to grid mesh length, $M .(\triangle)$ : case A, $(\diamond)$ : case $\mathrm{B},(\triangleright)$ : case $\mathrm{C}$ and $(\mathbf{\square})$ : case D.

FST cases. The definition of each of the parameters presented in this table are given in the following paragraphs.

There are several common approaches to define the thickness of the boundary layer. A common method is to report this thickness as the height at which the mean streamwise velocity in the boundary layer is equal to a fraction of the mean free-stream velocity, such as $\delta_{95}, \delta_{99}$ or $\delta_{995}$, corresponding to the height where the mean free-stream velocity reaches $95 \%, 99 \%$ or $99.5 \%$ of $U_{0}$, respectively. However, high fluctuations in the freestream due to the presence of the grid resulted in a wide scatter around the mean free-stream velocity which could lead to the uncertainty associated with identifying the boundary layer thickness using these definitions. For this reason the boundary layer thickness, $\delta$ was defined using an integral method, which was found to be a more robust method for this study. The boundary layer thickness is calculated using the iterative integral method given by Perry \& $\mathrm{Li}(1990)$ as $\delta=\delta^{*} U_{0} /\left(C_{1} U_{\tau}\right)$ where $\delta^{*}$ is the displacement thickness, $\delta^{*}=\int_{0}^{\infty}\left(1-\frac{U}{U_{0}}\right) d y, C_{1}=\int_{0}^{1}\left(\frac{U_{0}-U}{U_{\tau}}\right) d \frac{y}{\delta}$ and $U_{\tau}$ is the skin friction velocity obtained from Preston tube measurements. For a canonical boundary layer, this method results in typically $20 \%$ higher $\delta$ values than that defined using $\delta_{99}$ (Perry \& Li 1990).

Hancock \& Bradshaw (1983) introduced the FST parameter, $\beta$, defined as $\left(\sqrt{\overline{u_{0}^{2}}} / U_{0}\right) /\left(L_{e} / \delta_{995}+2\right)$ where the turbulence intensity $\left(\sqrt{\overline{u_{0}^{2}}} / U_{0}\right)$ is given as a percentage and $L_{e}$ is a turbulence length scale defined from the decay power law of the grid turbulence (Castro 1984). The purpose of this parameter is to account for the overall effect of FST by including the level of the turbulence and the characteristic length scale. Hancock \& Bradshaw (1983) had a $\beta$ range of 0.62 to 1.72, Thole \& Bogard (1996) had a range of 2 to 4.6 and Sharp et al. (2009) presented results with $\beta$ values of 1.5 and 2.3. It should be noted that Thole \& Bogard (1996) used the $\delta_{99}$ definition instead of $\delta_{995}$ for the boundary layer thickness. Likewise, the present study invokes a different definition of the thickness, i.e. $\delta$ definition given above, to calculate $\beta$ values presented in table 2. The next tabulated parameter $\Pi$ values represent the Coles wake parameter as defined by Coles (1956). Finally, the momentum thickness $\left(\theta=\int_{0}^{\infty} \frac{U}{U_{0}}\left(1-\frac{U}{U_{0}}\right) d y\right)$, 


$\begin{array}{ccccccccccc}\text { Cases } & & U_{0}(m / s) & \sqrt{\overline{u_{0}^{2}}} / U_{0}(\%) & \delta(m) & \beta & \Pi & U_{\tau}(m / s) & R e_{\tau} & R e_{\theta} & R e_{x} \\ \text { No-FST } & (\nabla) & 10.1 & 0.6 & 0.05 & - & 0.55 & 0.39 & 1330 & 3830 & 2.2 \times 10^{6} \\ \text { A } & (\triangle) & 6.1 & 7.4 & 0.11 & 1.1 & -0.26 & 0.27 & 1960 & 2760 & 1.5 \times 10^{6} \\ \text { B } & (\diamond) & 10.1 & 8.3 & 0.13 & 1.3 & -0.26 & 0.41 & 3450 & 4870 & 2.3 \times 10^{6} \\ \text { C } & (\triangleright) & 8.0 & 12.1 & 0.16 & 5.0 & -0.44 & 0.34 & 3670 & 4360 & 1.8 \times 10^{6} \\ \text { D } & (\square) & 9.9 & 12.7 & 0.17 & 4.9 & -0.52 & 0.42 & 4720 & 5590 & 2.3 \times 10^{6}\end{array}$

Table 2: (Colour online) Turbulent boundary layer parameters for FST cases at $x=$ $43 M$, with the baseline no-FST case. $U_{0}$ : mean streamwise velocity of the free-stream, $\sqrt{\overline{\overline{u_{0}^{2}}}} / U_{0}(\%)$ : free-stream turbulence intensity, $\delta$ : boundary layer thickness, $\beta$ : Hancock $\&$ Bradshaw (1983) FST parameter, $\Pi$ : Coles wake parameter, $U_{\tau}$ : skin friction velocity, $R e_{\tau}$ : Reynolds number based on skin friction velocity, $R e_{\theta}$ : Reynolds number based on momentum thickness, $R e_{x}$ : Reynolds number based on streamwise distance, $x$. Detailed definitions of the parameters are given in the text.

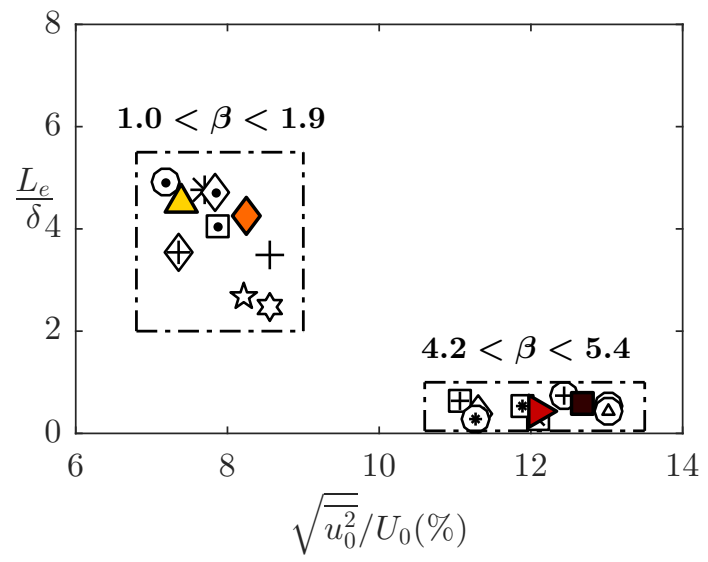

Figure 4: (Colour online) Turbulence length scale ratio defined by Hancock \& Bradshaw (1983) with FST turbulence level. $(\triangle)$ case A; $(\diamond)$ case B; $(\triangleright)$ case $\mathrm{C} ;(\boldsymbol{\square})$ case D. Conditions of the cases for all other data markers are found in table 3 in Appendix A.

skin-friction velocity and streamwise distance $(x)$ based Reynolds numbers are given in table 2 as $R e_{\theta}=U_{0} \theta / \nu, R e_{\tau}=U_{\tau} \delta / \nu$ and $R e_{x}=U_{0} x / \nu$, respectively.

A length scale ratio $\left(L_{e} / \delta\right)$, originally proposed by Hancock \& Bradshaw (1983) (albeit using $\left.\delta_{995}\right)$ is defined for the FST cases. Figure 4 shows this length scale ratio with respect to the free-stream turbulence intensity. This plot and subsequent parameter plots are populated with the inclusive dataset summarised by tables 2 and 3 to reveal the trends (or lack of) between the relevant parameters. Referring back to figure 4 , the cases can be grouped in two different $\beta$ ranges, which is mainly dependent on the wing type used. The four cases given in table 2 are chosen to be representative of these two distinct groups.

The extent of the parameter domain for $R e_{\lambda_{0}}, R e_{\tau}$ and $R e_{\theta}$ is shown in figure 5 . Referring to figure $5 \mathrm{a}, R e_{\tau}$ appears to linearly increase with $R e_{\lambda_{0}}$. It must be noted that 


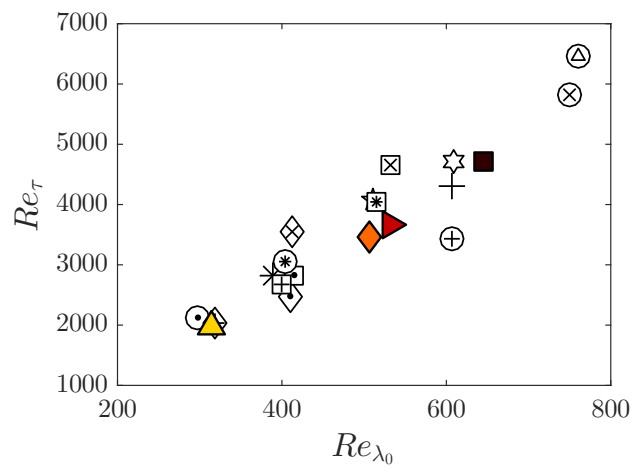

(a)

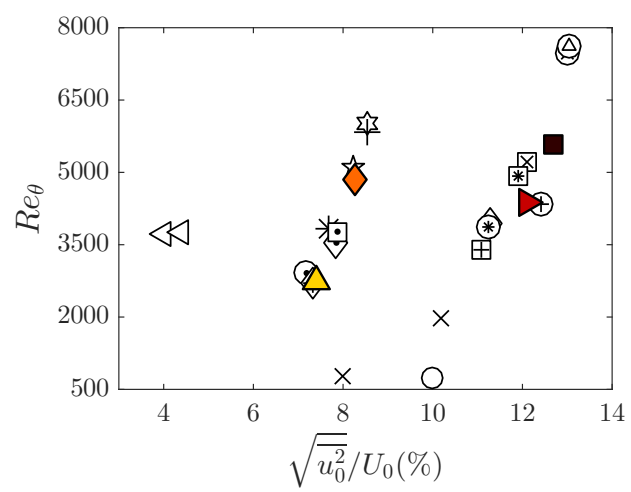

(b)

Figure 5: (Colour online) Parameter range. (a) $R e_{\tau}$ vs $R e_{\lambda_{0}}$; (b) $R e_{\theta}$ vs turbulence intensity level $\left(\sqrt{\overline{u_{0}^{2}}} / U_{0}(\%)\right) .(\triangle)$ case A; $(\diamond)$ case B; $(\triangleright)$ case C; $(\boldsymbol{\square})$ case D. Previous studies plotted in (b): $(\triangleleft)$ Hancock \& Bradshaw (1983), (○) Thole \& Bogard (1996), (x) Sharp et al. (2009). Conditions of the cases for all other data markers are found in table 3 in Appendix A.

although a distributed range of $R e_{\lambda_{0}}$ have been obtained in the experiments, the range of turbulence intensities that were achieved is more clustered. This is primarily because the turbulence intensity values were related to the solidity of the wings of the active grid, as previously discussed. However, the variation of the Taylor micro-scale appears to be dependent on the actuation scheme of the active grid. Regardless, the approximate linear relationship between $R e_{\lambda_{0}}$ and $R e_{\tau}$ is reasonably clear from this figure. The four cases chosen for this paper (table 2) also well manifest this linear relationship. It should also be noted that $R e_{\tau}$ range covered for the present study is not as wide as desired. Figure $5 \mathrm{~b}$ shows the relationship between turbulence intensity of the free-stream $\left(\sqrt{\overline{u_{0}^{2}}} / U_{0}(\%)\right)$ and $R e_{\theta}$. This figure shows that for each of the low- and high-range FST groups, $R e_{\theta}$ increases with the level of FST. However, comparing the two different groups, a similar range of $R e_{\theta}$ is observed although the level of FST is markedly different. This suggests that similar boundary layer characteristics may be developed under the effect of different FST levels and characteristics, which is further supported by the inclusion of several relevant examples from the literature. It can also be seen that the present study achieves higher $R_{\theta}$ values with higher turbulence levels compared to those previous studies.

The shape factor, $H=\delta^{*} / \theta$, is in relevance to the evolution of the boundary layer and is a measure of the "fullness" of the boundary layer. Due to penetration of FST and therefore mixing in the boundary layer, there is higher momentum flux towards the wall and this increases the fullness of the velocity profile. That in turn means a decrease in the shape factor, $H$, as also shown in various previous studies (Hancock \& Bradshaw 1983; Castro 1984; Stefes \& Fernholz 2004; Brzek et al. 2009). This is demonstrated in figure $6 \mathrm{a}$, which shows that the shape factor is reduced by the presence of FST in comparison to the no-FST case, which exhibits a typical value of around 1.4 for a turbulent boundary layer. Furthermore, the high-range turbulence intensity cases, i.e. cases with solid wings, typically have even lower values of $H$ compared to the low-range turbulence intensity cases, i.e. cases with cut-out wings. This suggests that there is higher FST penetration into the boundary layer for the higher turbulence intensity cases. The data of Hancock \& Bradshaw (1983), which has low turbulence intensity of around 3-4\% generated by a 


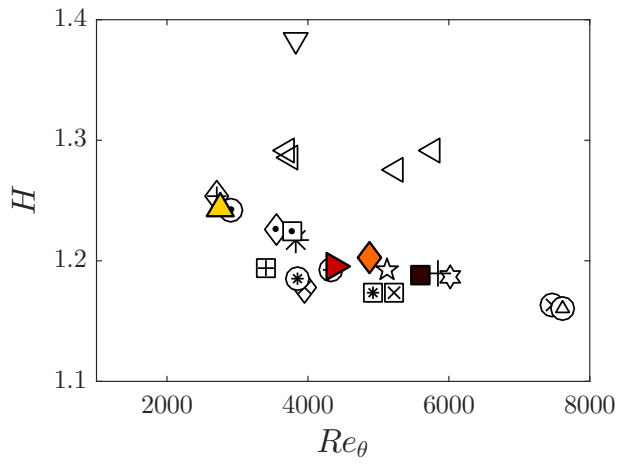

(a)

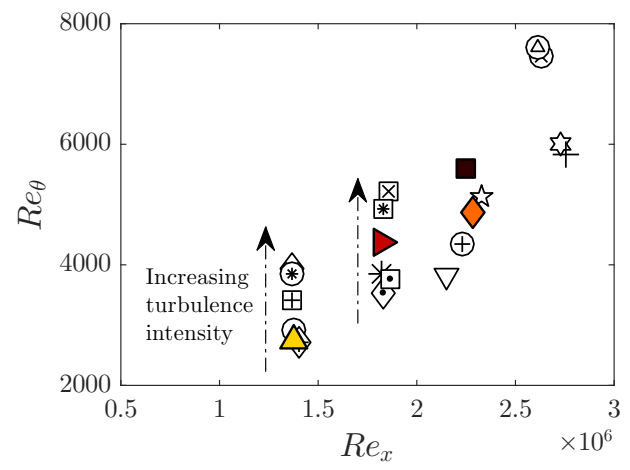

(b)

Figure 6: (Colour online) Development of boundary layer parameters. (a) Shape factor $(H)$ with $R e_{\theta}$; (b) $R e_{\theta}$ with $R e_{x} .(\triangle)$ case A; $(\diamond)$ case B; $(\triangleright)$ case C; $(\square)$ case D. No-FST case $(\nabla)$ and relevant data from Hancock \& Bradshaw (1983) $(\triangleleft)$ are also plotted in (a). Conditions of the cases for all other data markers are found in table 3 in Appendix A.

static grid, is also shown in figure $6 \mathrm{a}$. The shape factors of their cases reside between the no-FST case and those of the comparatively higher FST cases of the present study, which supports the systematical reduction of the shape factor with the increase in free-stream turbulence level. Furthermore, it is known that $H$ is a slowly decreasing function of $R e_{\theta}$ for a canonical turbulent boundary layer (Monkewitz et al. 2008) and this observation also appears to be valid for the FST cases shown in figure $6 \mathrm{a}$.

Thus far, we have shown data that demonstrates the variation of different boundary layer parameters with different free-stream turbulence conditions. The aforementioned results were obtained by altering the velocity of the free-stream, the active grid actuation scheme and its blockage. However, in order to examine the development of the boundary layer, it is perhaps more relevant to compare the state of the boundary layer at a fixed unit Reynolds number. In order to compare this, the boundary layer state (in this case $\left.R e_{\theta}\right)$ is plotted against the Reynolds number based on the downstream distance of the test plate, $R e_{x}$, in figure $6 \mathrm{~b}$. This representation takes into account the decay of the FST and the growth of the boundary layer in some ways and can be used to compare cases at the same $R e_{x}$ for different intensity values of FST. If the power-law decay rate of the FST is the same across different cases (as previously shown), then this plot shows the development of the boundary layer up to a fixed value of $R e_{x}$. The figure shows that for the same $R e_{x}$ (indicated by dashed arrows in the direction of increasing turbulence intensity), higher turbulence intensity in the free-stream results in higher $R e_{\theta}$. It can also be observed that one could change $R e_{x}$ and obtain the same $R e_{\theta}$ by modifying the free-stream turbulence intensity. It should be noted that the four FST cases given in table 2 enable the comparison of the effects of different FST levels on the boundary layer for a fixed $R e_{x}$, say cases $\mathrm{B}$ and $\mathrm{D}$, and the effects of different $R e_{x}$ for similar FST levels, say cases $\mathrm{A}$ and $\mathrm{B}$ and/or cases $\mathrm{C}$ and $\mathrm{D}$.

It has been well established that the presence of FST increases the skin friction in the boundary layer (Hancock \& Bradshaw 1983; Blair 1983; Castro 1984; Stefes \& Fernholz 2004). For a smooth wall zero-pressure gradient boundary layer without FST, the skin friction coefficient is given as a function of Reynolds number, $R e_{\theta}$, through a semiempirical relation by Fernholz \& Finley (1996). The baseline case from this study is captured by their relation, as shown in figure 7, whereas the FST cases result in larger 


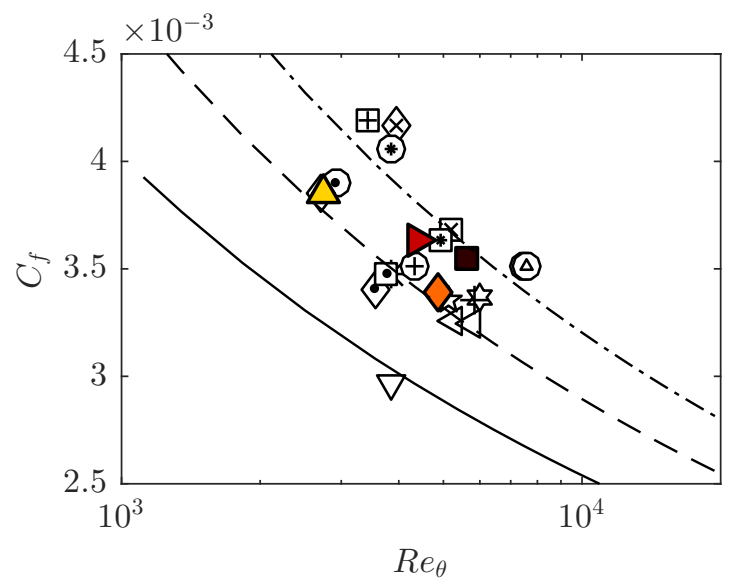

Figure 7: (Colour online) Variation of skin friction coefficient, $C_{f}$ with $R e_{\theta} .(\triangle)$ case A; $(\diamond)$ case B; $(\triangleright)$ case C; $(\square)$ case D; $(\nabla)$ no-FST case; $(\triangleleft)$ Hancock \& Bradshaw (1983); (-) Fernholz \& Finley (1996) correlation, (- -) fitted line for low range turbulence intensity cases and (-.) fitted line for high range turbulence intensity cases based on Fernholz \& Finley (1996) correlation. Conditions of the cases for all other data markers are found in table 3 in Appendix A.

values of the skin friction coefficient compared to this relation. Figure 7 also appears to show that the empirical relation of Fernholz \& Finley (1996) seems to hold for the FST cases, however, with different offset values for the two different turbulence ranges shown in the plot as fitted lines. At a constant $R e_{\theta}$ of, say, 6000 from figure 7 , where there is data for three different turbulence intensities (one of Hancock \& Bradshaw (1983) and two of present study cases of low- and high-range turbulence), it can be seen that an increase in the free-stream turbulence intensity causes an increase in the skin friction coefficient.

The preceding analysis demonstrated the significance of FST on properties of the boundary layer. We now consider outer-variable scaling of the free-stream velocity fluctuations since the boundary layer thickness and the FST appeared to be coupled (see table 2). Figure 8 shows the pre-multiplied energy spectra of the streamwise velocity fluctuations in the free-stream normalised by local free-stream variance, $k_{x} \phi_{u u} / \overline{u_{0}^{2}}$. The streamwise wavelength, $\zeta_{x}$, appears on the abscissa normalised by $\delta$. Each case appears to be forced from the free-stream at a similar length scale of around $10 \delta$. This suggests that the external disturbance experienced by the boundary layer is most energetic at about $10 \delta$ and the response to forcing at this dominant scale for different levels of forcing (i.e. FST level) can be examined in detail by comparing the boundary layer subjected to these different cases. The following sections will examine the influence of FST on the statistical as well as spectral characteristics of a turbulent boundary layer.

\subsection{Mean velocity}

The inner-normalised mean streamwise flow velocity is shown in figure 9 for the study cases together with the baseline case. The skin friction velocity in all cases was determined using the Preston tube method. A remarkable feature of the mean velocity profiles of turbulent boundary layers subjected to FST is the persistence of the log-law region for FST levels up to 20\% (Thole \& Bogard 1996). The inner-scaled profiles (figure 9) here also confirm the validity of the log-law. It shows a clear collapse below the outer region 


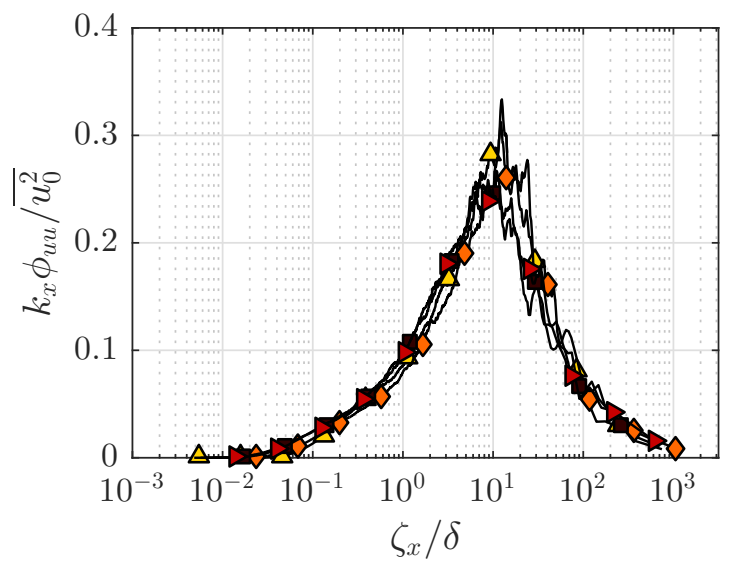

Figure 8: (Colour online) Normalised pre-multiplied energy spectra of streamwise velocity fluctuations, $k_{x} \phi_{u u} \overline{u_{0}^{2}}$, of the free-stream for the FST cases with streamwise wavelength, $\zeta_{x}$, of the free-stream with respect to boundary layer thickness, $\delta .(\triangle)$ case A; $(\diamond)$ case B; $(\triangleright)$ case C; $(\mathbf{\square})$ case D.

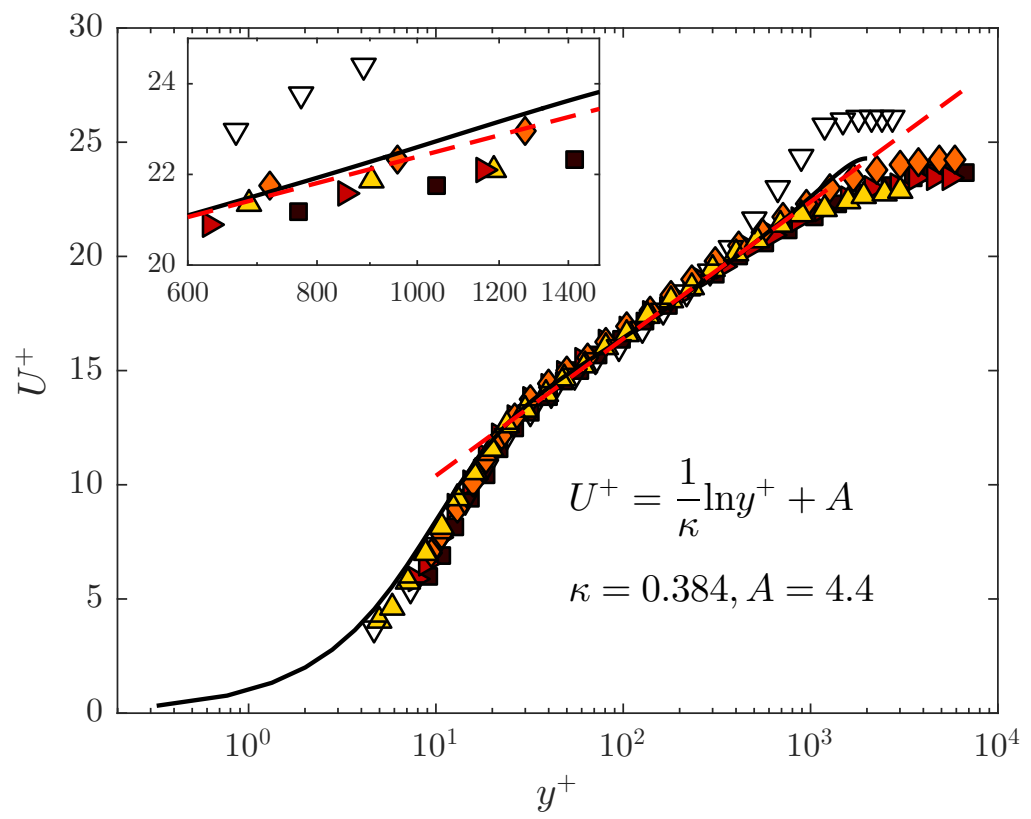

Figure 9: (Colour online) Inner-normalised mean velocity profiles. $(\triangle)$ case $A ;(\diamond)$ case B; $(\triangleright)$ case C; $(\boldsymbol{\square})$ case D; $(\nabla)$ no-FST case; dashed line: log-law with coefficients $\kappa=0.384$ and $A=4.4$, solid line: DNS channel flow data at $R e_{\tau} \approx 2000$ from Hoyas \& Jiménez (2006). The inset shows a close-up of the profiles for the range of $600<y^{+}<1500$. 
with log-law coefficients of von Kármán constant, $\kappa=0.384$ and constant, $A=4.4$; these coefficient values follow from Marusic et al. (2013). This suggests that the effect of FST on the mean flow in the inner region is to alter the value of skin-friction velocity. However, once this change is accounted for, there is self-similarity in the logarithmic region and below. This indicates a proportional effect of the FST in the mean flow and robustness of the law-of-the-wall even when subjected to large external disturbances. This collapse in the mean profiles has also been interpreted by Hancock \& Bradshaw (1989) as the mean flow being less affected by FST. The mean velocity profiles deviate from the similarity to the baseline no-FST case as the FST cases have an extended logarithmic region compared to the no-FST case. From the figure, the deviation point of the profiles from the log-law is obvious for no-FST case whereas for the FST cases deviation from that curve occurs at much higher wall-normal positions (in wall units); therefore implying an extended region that is defined by the logarithmic law. The inset in the figure shows a close-up view of the profiles which highlights the similar extension of the logarithmic region for the FST cases. Within the measurement uncertainty, the profiles exhibit a logarithmic region that extends up to $y^{+} \approx 700$. This has important implications for the overlap region argument of the turbulent boundary layer. It can be deduced that the validity of the law of the wall, i.e. $U^{+}=f\left(y^{+}\right)$, is not dictated here by the outer scales in the boundary layer and FST can be said to affect these outer scales in such a way that the law of the wall can extend up to higher wall-normal positions in wall units. It is assumed for a canonical turbulent boundary layer that the logarithmic law is valid up to $y^{+}=0.2 R e_{\tau}$; therefore looking at the extended $y^{+}$values from the figure, a Reynolds number analogy between the present FST cases and canonical boundary layers can be made. This would imply for the present FST cases to represent a high Reynolds number flow without FST at $R e_{\tau}$ of around 3500 .

The extension of the logarithmic region is intimately connected to the suppression of the wake region under the effect of FST. In a canonical boundary layer like the no-FST case, the profile is bounded by the onset of intermittency resulting in a well-defined wake region; however for a turbulent boundary layer under the FST effect, there is no intermittent region since the free-stream is also turbulent and thus the wake region is almost vanished. For this reason, turbulent boundary layers under the FST effect can be said to have similarities with a turbulent channel flow where there is significant turbulence in the outer region at the centreline of the channel. Direct numerical simulation (DNS) data of a turbulent channel flow at $R e_{\tau} \approx 2000$ from Hoyas \& Jiménez (2006) has been added on the mean profile plot for comparison. The suppression of the wake region with respect to the no-FST case is also obvious from their data and shows similarities to the present FST cases. This lack of wake in channel is primarily due to the presence of a turbulent core. In addition to turbulent core, the presence of favourable pressure gradient (FPG) in channel flows would also tend to suppress the wake region (Nagib \& Chauhan 2008); however, this pressure gradient effect is likely to be weak especially at higher Reynolds number. Having said that, presence of FPG will indeed reduce the wake strength in external flow (i.e. boundary layers) due to the acceleration of the free-stream. In fact, Stefes \& Fernholz (2004) made an analogy between the FST effect on the wake region of the turbulent boundary layer and the effect of a favourable pressure gradient on a turbulent boundary layer. Consequently, external flows with FPG such as sink flows (Jones et al. 2001) can also produce a mean flow that is similar to the ones presented in the current study, however, the turbulence properties are likely to be different.

The velocity defect profiles in two different scalings are given in figure 10 . The suppression of the wake region can be clearly observed from both scalings. For the first scaling shown in figure 10a, the velocity defect is either very little or absent in the 


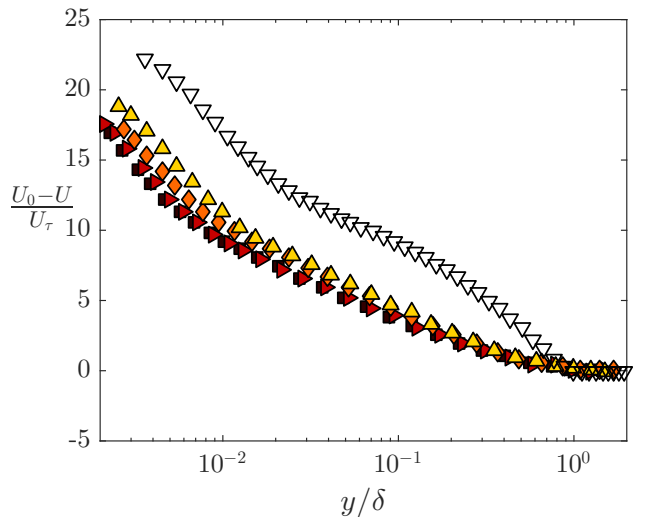

(a)

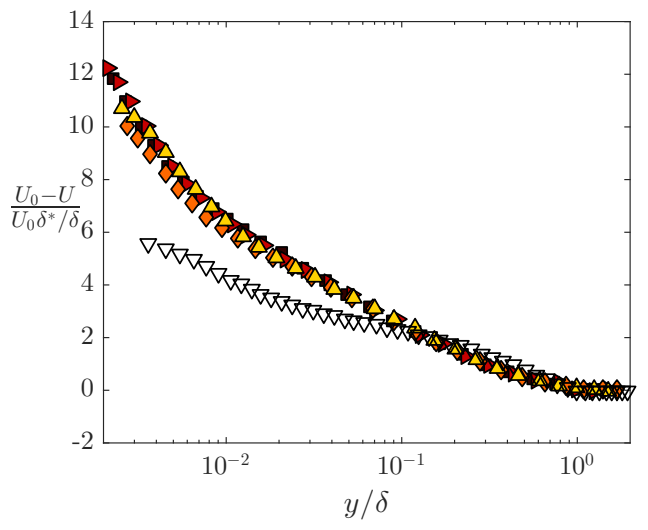

(b)

Figure 10: (Colour online) Velocity defect profiles normalised by (a) inner velocity scaling (b) outer velocity scaling proposed by Zagarola \& Smits $(1998) .(\triangle)$ case A; $(\diamond)$ case B; $(\triangleright)$ case $\mathrm{C}$; $(\mathbf{\square})$ case $\mathrm{D} ;(\nabla)$ no-FST case.

outer region of the boundary layer when compared with the no-FST case. This results in reduced wake strength as expected for a turbulent boundary layer under the effect of FST (Thole \& Bogard 1996; Sharp et al. 2009). Correspondingly, the Coles wake parameter, $\Pi$, that quantifies the wake strength reduces with the presence of FST and even becomes negative for strong FST cases as shown in table 2. In figure 10b, the outer velocity scale proposed by Zagarola \& Smits (1998) is considered following the observations by Brzek et al. (2009). Although the scaling effectively removes Reynolds number variations in the mean velocity defect law for various flows (Klewicki 2010), it has been shown by Brzek et al. (2009) that it does not completely eliminate the effects of high FST. Therefore, the Zagarola and Smits scaling does not offer any improvement compared to inner-scaling for the velocity-defect similarity of the FST cases with respect to the no-FST case; however, the various FST cases appear to collapse. This scaling is proportional to the mass flux deficit, i.e. $\delta^{*}$, and since the mass flux deficit does not vary among different FST cases, the similarities between different cases can therefore be justified. Although Brzek et al. (2009) observed an improved collapse with the Zagarola and Smits scaling, an overall collapse as shown in figure $10 \mathrm{~b}$ was not achieved. This is probably due to the fact that the mass flux in their case is also under the influence of surface roughness.

\subsection{Streamwise broadband turbulence intensity and energy spectra}

The broadband turbulence intensity, i.e. variance, profiles of the streamwise velocity fluctuations are given in figure 11 for two different normalisations. For the first case, the variance profiles are normalised by corresponding variance of the free-stream fluctuations (figure 11a). Within the boundary layer the normalisation does not collapse these profiles. Rather, this normalisation may be interpreted as a gain of the turbulence level in the boundary layer compared to the free-stream turbulence whereby the turbulent fluctuations outside the boundary layer amplify the fluctuations within the boundary layer. Therefore, in the free-stream the amplification is exactly $\overline{u^{2}} / \overline{u_{0}^{2}}=1$. Within the boundary layer the amplification may vary up to the wall owing to the penetration of FST. Using this analogy, we can further say that the level of amplification and the distribution varies with the level of the free-stream disturbance. For example, it is shown in figure 11a that for the higher turbulence levels a more uniform amplification throughout the 


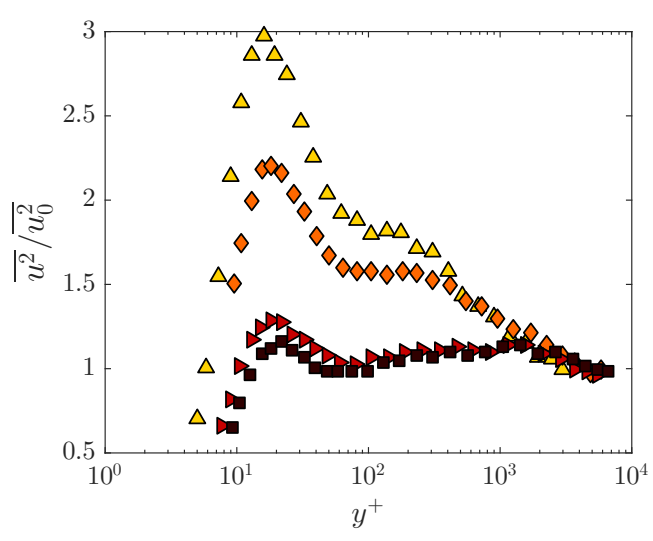

(a)

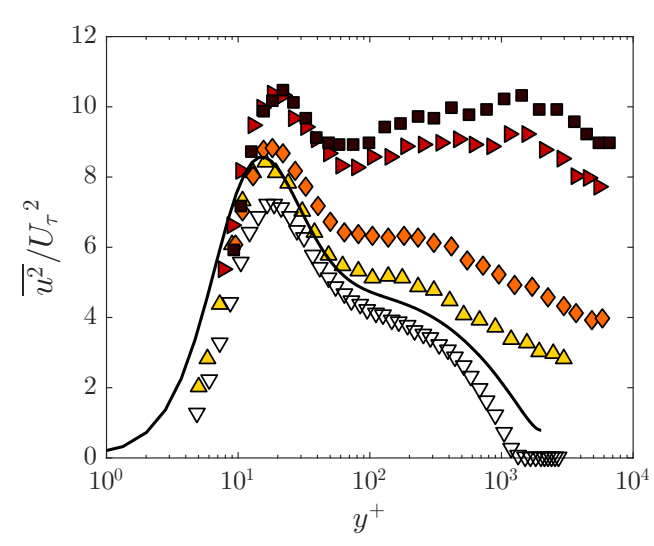

(b)

Figure 11: (Colour online) Variance profiles of streamwise velocity fluctuations. (a) Freestream variance normalised; (b) inner-normalised. $(\triangle)$ case A; $(\diamond)$ case B; $(\triangleright)$ case C; $(\square)$ case D. No-FST case $(\nabla)$ and DNS channel flow data at $R e_{\tau} \approx 2000$ from Hoyas \& Jiménez (2006) (solid line) are also plotted in (b).

boundary layer exists. In contrast, for lower turbulence range, the near-wall turbulence peak remains a dominant feature. Therefore, the highest amplification likely occurs when the free-stream is laminar and when the fluctuations in the near-wall are solely governed by near-wall cycle. This suggests a possible saturation limit for the turbulence within the boundary layer when subjected to external disturbances. Because a relative effect of the FST at each wall normal location can be observed, the investigation of the energy distribution throughout the boundary layer with spectral analysis will be used to clarify how the energy of the FST affects the different regions of the boundary layer.

The inner-normalised variance profiles are presented in figure $11 \mathrm{~b}$ in comparison with the no-FST case and with the DNS data from Hoyas \& Jiménez (2006) for a turbulent channel flow. The response of the fluctuating component of the streamwise velocity in the boundary layer to the change in the free-stream turbulence level is not consistent, which is similarly found in figure 11a. Immediately evident from figure 11b are the behaviour of the near-wall peak and the emergence of an outer-peak for these laboratory scale flows. In the following sections, we examine both these features in greater detail.

\subsubsection{Near-wall region}

For canonical turbulent boundary layers, the near-wall turbulence production, governed by the near-wall cycle of streaks and quasi-streamwise vortices, peaks at $y^{+} \approx 15$ as documented in many studies to date (Smits et al. 2011). In the present study, the location of the near-wall peak appears to propagate away from the wall (for inner scaling) with increasing Reynolds number as shown in figure 12a for the inclusive dataset summarised in tables 2 and 3. The error bars represent the largest uncertainty in locating the wall to justify the discrepancy of the present cases from the expected $y^{+} \approx 15$ location. The outward movement of the near-wall peak, in wall units, has important implications in terms of the extent of the penetration and the governance of the near-wall turbulence production. Similar behaviour can be found in the comparable data shown by Stefes \& Fernholz (2004); however, they did not mention this gradual movement of the peak location. Regardless, the current study does not have sufficient range of conditions to identify a definitive relationship between FST and the movement of the near-wall peak. 


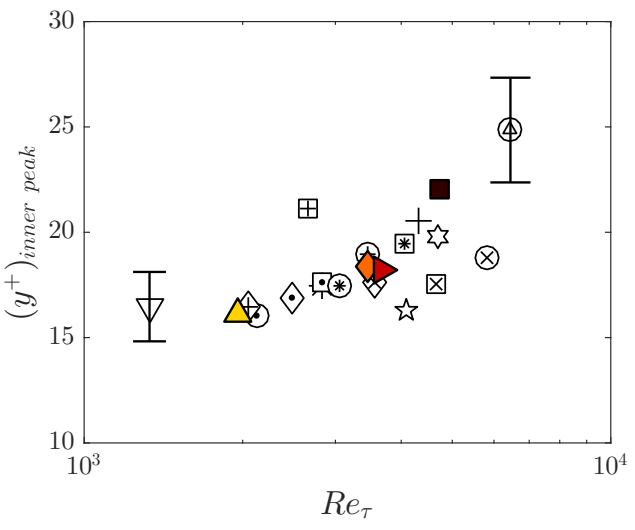

(a)

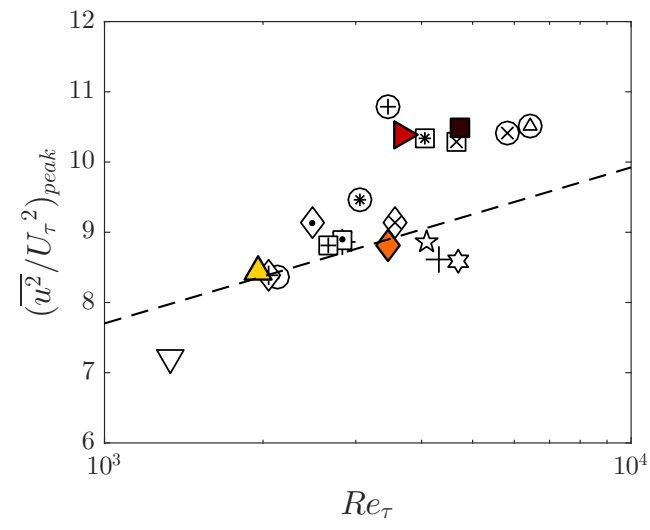

(b)

Figure 12: (Colour online) Variation of (a) the location and (b) the amplitude of the near-wall peak of the inner-normalised variance of the streamwise velocity fluctuations with Reynolds number. $(\triangle)$ case A; $(\diamond)$ case B; $(\triangleright)$ case C; $(\boldsymbol{\square})$ case D; $(\nabla)$ no-FST case. Conditions of the cases for all other data markers are found in table 3 in Appendix A. Error bars shown in (a) correspond to $10 \%$ uncertainty in $y^{+}$, dashed line in (b) is the curve fit, $\left(\overline{u^{2}} / U_{\tau}^{2}\right)_{\text {peak }}=1.036+0.965 \ln \left(R e_{\tau}\right)$, given by Hutchins \& Marusic $(2007 b)$.

The near-wall peak amplitude increases with the turbulence intensity level of the freestream. Similar attributes in the near-wall peak are also encountered in high Reynolds number boundary layers for increasing Reynolds numbers, $R e_{\tau}$ (Smits et al. 2011). For these type of flows, the energy in the outer region grows as the Reynolds number increases inferring that the fluctuations of the outer region structures are becoming stronger and comparable to the fluctuations of the near-wall structures (Hutchins \& Marusic 2007a). This induces a growing influence of the energetic outer region on the near-wall region and explains the increase in the near-wall peak amplitude. A possible analogy with high Reynolds number flows in this respect implies similar interactions between the structures of the near-wall and the large structures in the outer region for the present FST cases. The difference here in this study compared to a high Reynolds number flow without FST is that the outer large scales here are introduced by large scales of the free-stream that penetrate into the boundary layer. The trend in the near-wall peak amplitude for the FST cases is given in figure $12 \mathrm{~b}$, which is again populated with the inclusive dataset summarised in tables 2 and 3. Also included is the relation given by Hutchins \& Marusic $(2007 b)$ for the variation of the near-wall peak with $R e_{\tau}$. For low-range turbulence intensity cases, the data appears scattered about the relationship given by Hutchins \& Marusic $(2007 b)$ whereas the high-range turbulence intensity cases appear consistently above that relation. Regardless, it is evident that for FST cases, like in high Reynolds number flows, the increase in the near-wall peak can also be attributed to the increasing influence of the large scales on the near-wall region as they become more energetic with increasing Reynolds number.

It is also worth to mention that the increase in the fluctuation levels in the near-wall peak varies among the different cases. The difference in the peak levels with respect to the no-FST case is more prominent between the low- and high-range turbulence intensity cases (e.g. between case B and case D or between case A and case C) than between the similar intensity cases (e.g. between case A and case B or between case C and case D). 
This is due to higher penetration of FST into the boundary layer in higher FST cases, a point which will be made clear from examining the spectra. This also indicates that the increase in the level of fluctuations in the near-wall region is mostly governed by the level of turbulence in the free-stream.

Figure 11b also shows that the near-wall peak for case $\mathrm{A}$ is similar to that of a turbulent channel flow at a comparable Reynolds number. However, the channel flow has a freestream turbulence of only about $2 \%$ (i.e. the streamwise turbulence intensity at the centreline of the channel). This indicates that although the FST cases do not have an outer wake region in the mean flow due to loss of intermittency, analogous to the channel flow, a much higher free-stream turbulence appears to be necessary for the near-wall peak to have the same intensity as a channel flow at the same $R e_{\tau}$. This suggests that the near-wall region of the boundary layer damps out the outer disturbances much more than in a channel flow.

\subsubsection{Outer region}

The outer region of the boundary layer is directly affected by the presence of FST, in comparison to that of the near-wall region, as shown in figure $11 \mathrm{~b}$. The variance of the no-FST case levels off at approximately zero since the free-stream has near-zero turbulence whereas the FST cases level off at the non-zero free-stream fluctuation values. For each of the FST cases, starting from the level of free-stream turbulence at the edge of the boundary layer, the fluctuations in the outer region are amplified. The amplification there appears higher for high-range turbulence intensity cases. This reflects the growing energy level in the free-stream. Similarly, in high Reynolds number flows, the outer scales become more energetic as the Reynolds number increases and this is reflected in the emergence of an outer peak in the streamwise velocity spectra (Hutchins \& Marusic $2007 a$ ). Also, the increasing magnitude and constancy of the plateau-like region in the variance profiles is observed to coincide with that peak (Klewicki 2010). Therefore, in an attempt to investigate the energy distribution for the present cases, spectral analysis is performed and interesting similarities with high Reynolds number flows are observed.

Figure 13 shows contour maps of the inner-normalised pre-multiplied energy spectra of the streamwise velocity fluctuations that are plotted against wall distance, $y$, and streamwise wavelength, $\zeta_{x}\left(=2 \pi / k_{x}\right)$, for the FST cases with their corresponding mean velocity and variance profiles. Figure 13a to $13 \mathrm{~d}$ are in order of increasing turbulence intensity levels. They are also in the increasing order of friction Reynolds numbers with $R e_{\tau}=1960,3450,3670$ and 4720, respectively. For each of the contour maps two distinct peaks in the energy are observed, which will be referred to as the inner and outer spectral peaks and indicated by black and white $(+)$ symbols, respectively. The inner spectral peak corresponds to the near-wall cycle of streaks and quasi-streamwise vortices. The location of that peak is found to be fixed in inner coordinates at $y^{+} \approx 15$ and $\zeta_{x}^{+} \approx 1000$ from all the spectra plots as also previously found in Hutchins \& Marusic $(2007 a, b)$ for a canonical turbulent boundary layer. The vertical dashed lines following the inner spectral peak also shows the corresponding wall normal location in variance profiles presented in plot (ii) for each case. As FST level increases, it can be seen that the near-wall spectral peak remains rooted at $y^{+}=15$. However, the near-wall broadband peak in the variance seems to move outwards as observed previously in figure 12a. The disparity between these two peak locations can be related to the low wavenumber content of the FST in the near-wall region. The footprint of the outer broadband peak in the near-wall is essentially causing the inner broadband peak to occur further away from the wall. Apart from indicating how far the penetration can go down to very near-wall, it can also be deduced that the extent 


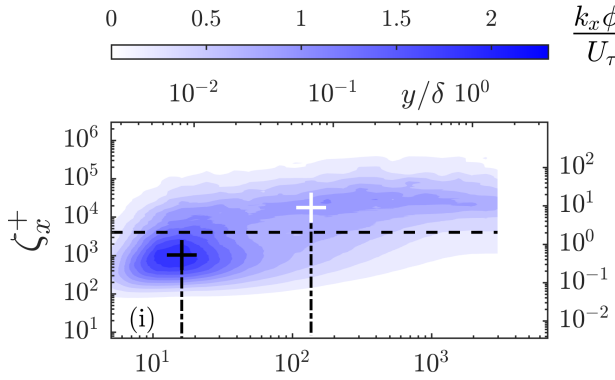

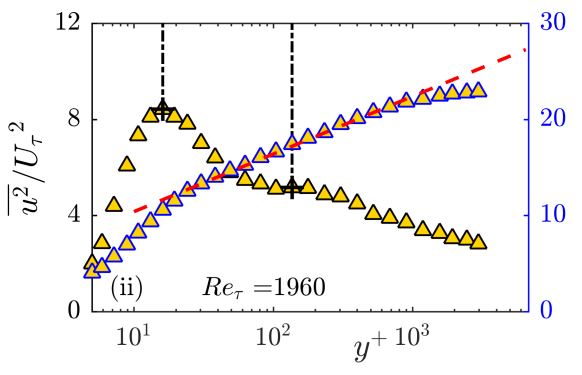

(a)
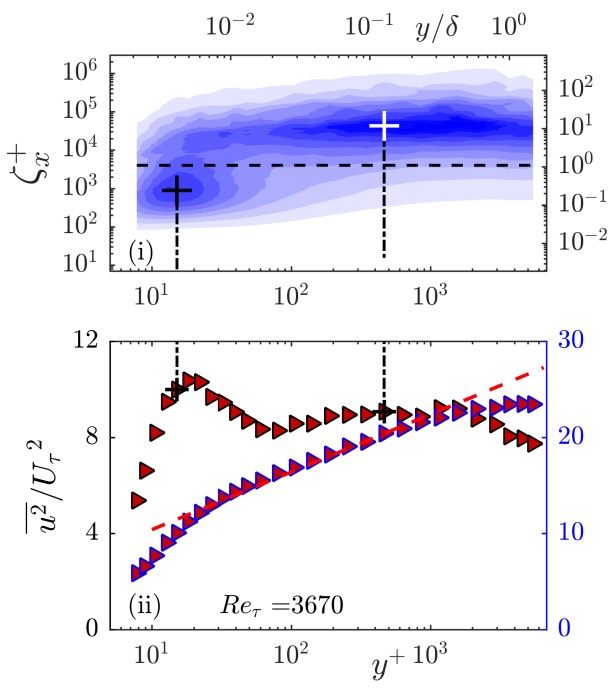

(c)
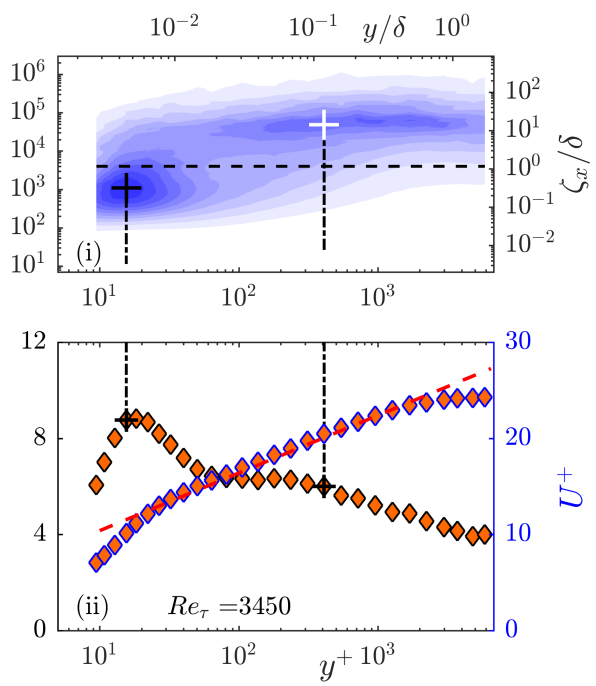

(b)
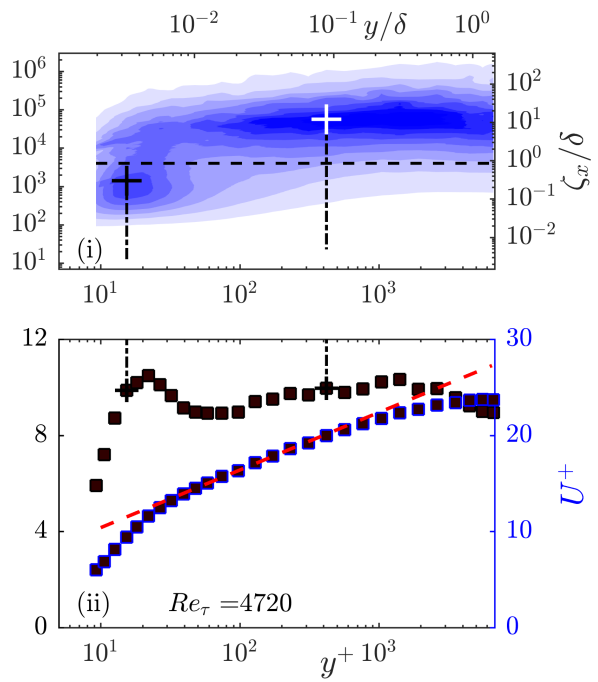

(d)

Figure 13: (Colour online) (i) Contour maps of the inner-normalised pre-multiplied energy spectra of the streamwise velocity fluctuations, $k_{x} \phi_{u u} / U_{\tau}^{2}$, for cases (a) $(\triangle)$ case A; (b) $(\diamond)$ case B; (c) $(\nabla)$ case C; (d) ( $\mathbf{\square})$ case D. The ordinates show streamwise wavelength, $\zeta_{x}$, in both inner (left) and outer (right) scaling. The abscissas show the wall normal location, $y$, also plotted in both inner (bottom) and outer (top) scaling. (+) indicates inner (black) and outer (white) spectral peaks. The horizontal dashed line represents the location of the cut-off wavelength at $\zeta_{x}^{+} \approx 4000$. (ii) Corresponding mean (blue outlined marker) and variance profiles. Dashed red line: log-law with coefficients $\kappa=0.384$ and $A=4.4$. Dot-dashed vertical lines and $(+)$ symbols represent the locations corresponding to the spectral peaks indicated on (i). 
of penetration depends on the level of turbulence. This is consistent with the observed increase in the near-wall peak amplitude (figure 11b) with increasing FST level.

As mentioned previously, there is a second distinct spectral peak in the outer region indicated with a white $(+)$ symbol on spectra plots. The emergent outer spectral peaks for FST cases seem to increase in magnitude with increasing Reynolds number, i.e. with increasing FST levels. A similar behaviour is also known to occur for the canonical turbulent boundary layer with increasing Reynolds number. This similarity could have implications in mimicking high Reynolds number flows that are difficult to achieve without a purpose-built facility, by using FST with much lower Reynolds numbers.

For the high-range turbulence cases, two distinct broadband peaks are observed in the variance profiles; here, however, we focus on the peak that corresponds to a location inside the logarithmic region. The origin of the other broadband peak further from the wall would require a large database of high FST cases to give detailed consideration and it is therefore not discussed here. Within the logarithmic region, the broadband turbulence peak coincides with plateau-like region in the variance profiles. For sufficiently high Reynolds number flows, i.e. having sufficient scale separation, the wall-normal location of that peak has been shown to nominally coincide with the centre of the log region as shown by Hutchins \& Marusic $(2007 a)$. They also related the outer peak with the large-scale structures in the log region. For FST cases, a similar behaviour is observed where there appears to be a nominal spectral peak in the log region. Furthermore, the peak energy in this region occurs about a wavelength of $10 \delta$, which is also the most energetic wavelength in the free-stream. This behaviour was also observed by Sharp et al. (2009) where they presented the pre-multiplied energy spectra of boundary layer at different wall-normal locations and the free-stream together and they concluded that the energetic wavelengths in the outer region were associated with the most energetic scales of the free-stream. Given the relationship between the free-stream energetic scales and the outer spectral peak, we can have a look at the ratio of their corresponding spectral energy at the outer peak wavelength. In this way, we could obtain a wavelength-specific gain of the turbulence level compared to FST as given in figure 14a. This is similar to the broadband gain that is previously presented in figure 11a. Likewise, it is deduced that the level of amplification varies with the level of free-stream and high-range FST cases tend to amplify less.

For canonical turbulent boundary layers, the location of the outer spectral peak has been shown to gradually move outward in wall units, $y^{+}$, with increasing $R e_{\tau}$ and this movement is also found to be proportional to $R e_{\tau}^{1 / 2}$ (Klewicki et al. 2007; Mathis et al. 2009). The location of the outer spectral peak for the inclusive set of FST cases show agreement with this trend as plotted in figure 14b. Despite the good agreement, it should be noted that the range of $R e_{\tau}$ covered in this study is limited and it is therefore very difficult to decipher this relationship without the guidance from previous work in this area.

\subsection{Scale separation and amplitude modulation}

Despite not having measurements very close to the wall, Sharp et al. (2009) suggested that the FST can reach deep into the boundary layer and have a direct effect on the inner scales of the boundary layer. The previous section demonstrated that the direct effect indeed reaches down to the wall and in fact moves the near-wall peak farther away from the wall. It is perhaps also important to examine any other effect of FST on nearwall turbulence. Decomposing the scales as large and small scales using a sharp spectral cut-off filter, Mathis et al. (2009) found that the near-wall turbulence is modulated by the energetic outer region of the flow in a canonical high Reynolds number boundary 


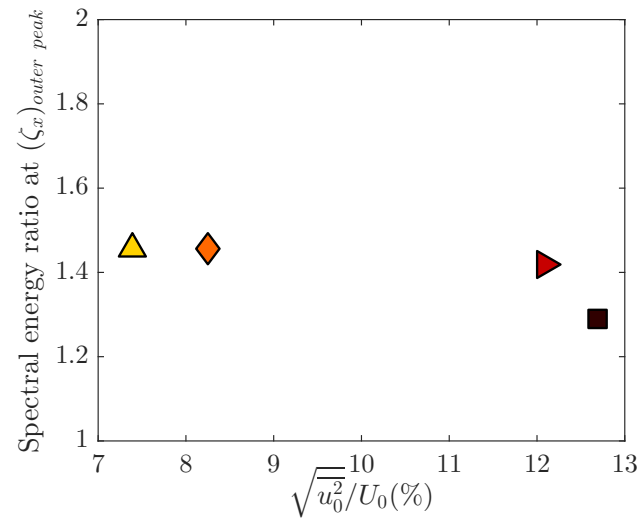

(a)

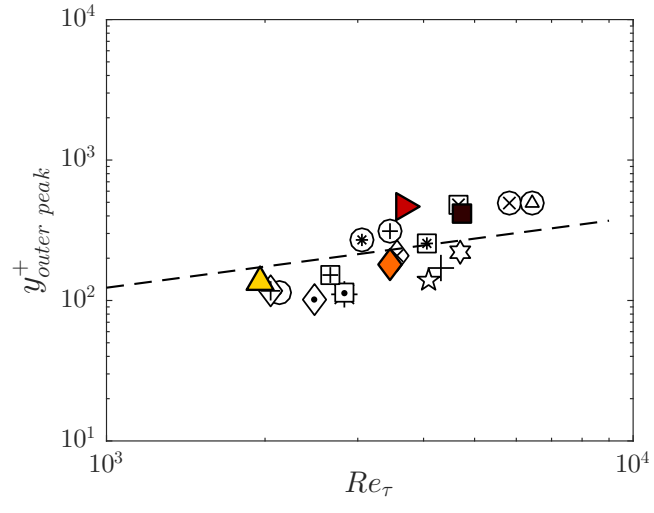

(b)

Figure 14: (Colour online) (a) Spectral energy ratio between the FST and the boundary layer at the outer peak wavelength (b) variation of the wall normal location of the outer peak of the inner-normalised variance of the streamwise velocity fluctuations with Reynolds number. $(\triangle)$ case A; $(\diamond)$ case B; $(\nabla)$ case C; $(\boldsymbol{\square})$ case D. Conditions of the cases for all other data markers are found in table 3 in Appendix A. Dashed line represents the relation of $3.9 R e_{\tau}^{1 / 2}$ from Mathis et al. (2009) for the wall-normal location of the outer peak based on the definition of the log region as $100<y^{+}<0.15 R e_{\tau}$.

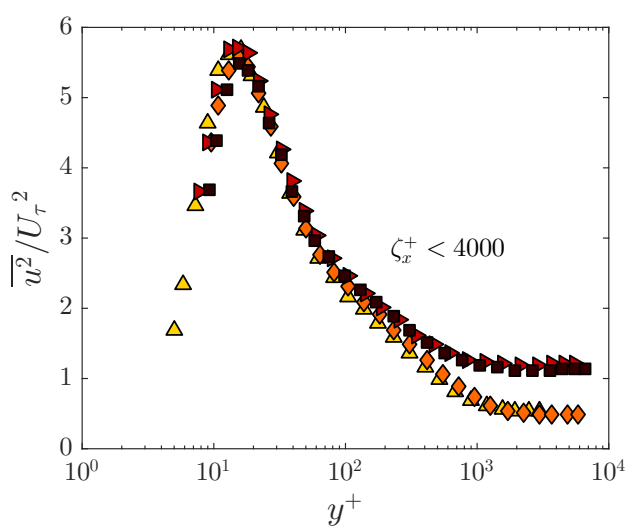

(a)

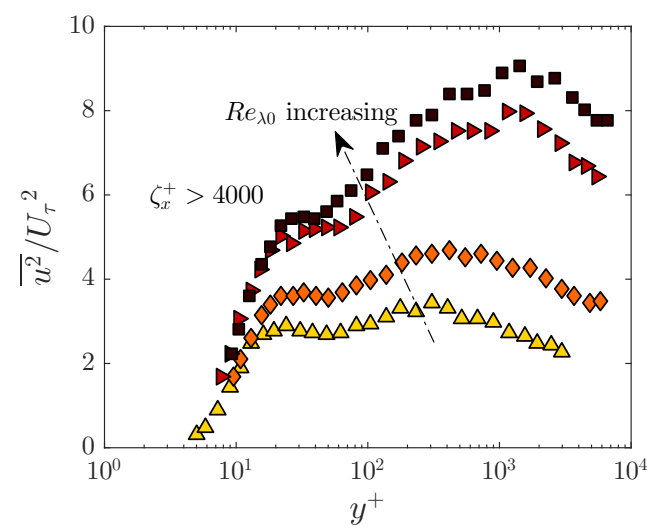

(b)

Figure 15: (Colour online) Variance profiles of scale-decomposed streamwise velocity fluctuations. (a) Small-scales and (b) large-scales based on a cut-off wavelength filter of

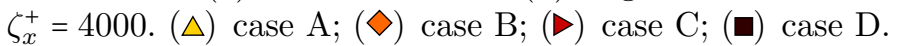

layer. We aim to carry out a similar analysis for the FST cases. A filter at a cut-off wavelength $\left(\zeta_{x}^{+}\right)$of 4000 (the horizontal dashed line in figure 13) represents a reasonable demarcation between the large- and small-scale components of the streamwise fluctuating velocity signal. This also corresponds to $\zeta_{x} / \delta \approx 1-2$ for all the cases. The robustness of the decoupling procedure is shown in Mathis et al. (2009) for various different cut-off wavelengths; therefore in terms of investigating the interactions between the large and small scales, the choice of cut-off wavelength is not expected to have a considerable effect. 
Figure 15 shows the inner-normalised variance profiles of scale-decomposed streamwise velocity fluctuations. These two separate variance profiles can be considered as the two components that add up to the variance profiles presented in figure $11 \mathrm{~b}$. The contribution from the small-scale to the total variance of the streamwise velocity fluctuations (figure 15a) does not seem to change with different turbulence intensity levels across most of the boundary layer; however, overall, the effect of small-scales seems to diminish to the edge of the boundary layer for all FST cases. The small-scale variance profiles have essentially eliminated any superposed effect of large-scale motions on the measured statistics. The fact that the small-scale contributions collapse for different cases after filtering out the superposition of large-scales indeed confirms the direct penetration effect of FST on the near-wall region as also suggested by Sharp et al. (2009).

The large-scale variance profiles exhibit an increase in the near-wall region, reaching a local maxima $\left(y^{+} \approx 15-30\right)$ and increases further farther away from the wall. Overall, large-scale contribution increases across almost all wall-normal locations with increasing FST levels. Apart from gradual movement of the local peak turbulence intensity location, there is also amplitude increase of the local peak with increasing FST levels. This is also observed in high Reynolds number flows as the increasing effect of large-scales on the near-wall region with increasing Reynolds number and this is found to be an indication of a growing outer-layer influence on the near-wall region (Hutchins \& Marusic 2007b; Smits et al. 2011).

Any modulation effect involved in the interactions between the scales is not directly inferred from these two plots as the modulation can be symmetric. More insights about the modulating effect of the large scales could be obtained from skewness analysis. Mathis et al. (2011b) confirmed that the amplitude modulation coefficient $(R)$ defined by Mathis et al. (2009) as the correlation coefficient between the large-scale fluctuations and the filtered envelope of the small-scale fluctuations is similar to the cross-term of the scaledecomposed skewness, $3 \overline{\overline{u_{L}^{+} u_{S}^{+2}}}$. They even suggested to alternatively use this cross-term to quantify the level of amplitude modulation instead of the amplitude modulation coefficient. Therefore, in the present study, the cross-term of the scale-decomposed skewness factor will be used to diagnose the modulating effects of the large scales. In order to do this, the expansion of $S_{u}$, as first used by Schlatter \& Örlü (2010) for wall-bounded flows and as outlined in Mathis et al. $(2011 b)$, is followed. Using a scale-decomposed signal of the fluctuating streamwise velocity signal as $u^{+}=u_{L}^{+}+u_{S}^{+}, S_{u}$ can be expanded in the following form

$$
S_{u}=\overline{\overline{u_{L}^{+3}}}+3 \overline{\overline{u_{L}^{+2} u_{S}^{+}}}+3 \overline{\overline{u_{L}^{+} u_{S}^{+2}}}+\overline{\overline{u_{S}^{+3}}}
$$

with $\overline{\bar{X}}=\bar{X} /\left(\overline{u^{+2}}\right)^{3 / 2}$ for any quantity X (refer to equation 2 to 4 in Mathis et al. $2011 b$ ).

Figure 16 shows the total skewness and the three dominant terms (terms 1, 3 and 4 in the above equation) that contribute to this skewness for different FST cases. The cross-term $3 \overline{\overline{u_{L}^{+2} u_{S}^{+}}}$is close to zero and does not contribute to the skewness factor and hence is not shown.

The total skewness factor is compared in figure 16a with the results of Mathis et al. $(2011 b)$ that was obtained from a turbulent boundary layer without FST at $R e_{\tau}=2800$. It is seen from this figure that the skewness profile of case A being the lowest turbulence intensity case with $R e_{\tau}=1960$ has closer values to their study. The similarity of the trend in the profiles starts to fail due to the presence of an intermittent region in their case of a turbulent boundary layer without FST whereas this intermittent region does not exist in a turbulent boundary layer under the FST effects. For turbulent boundary 


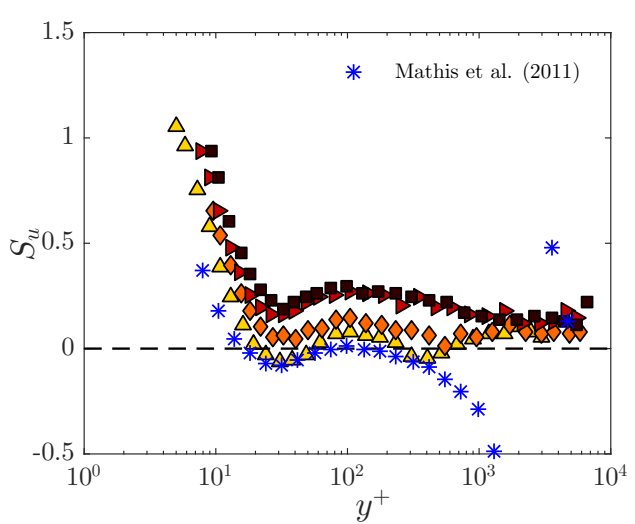

(a)

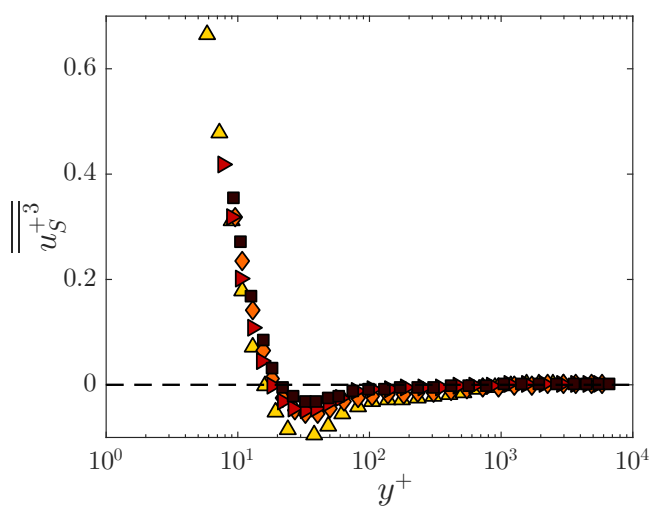

(c)

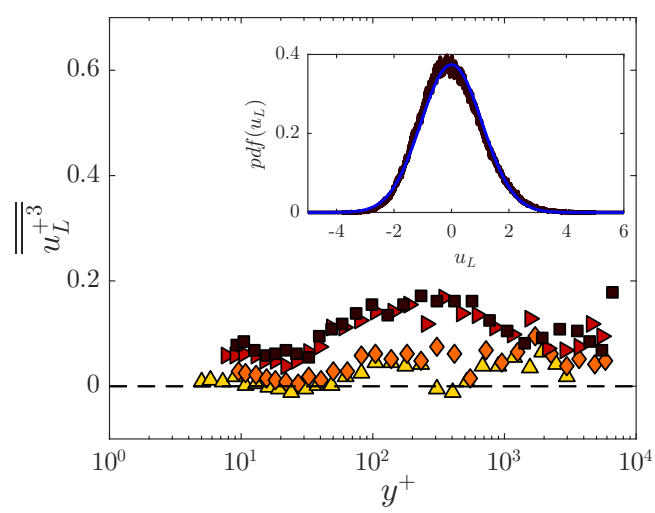

(b)

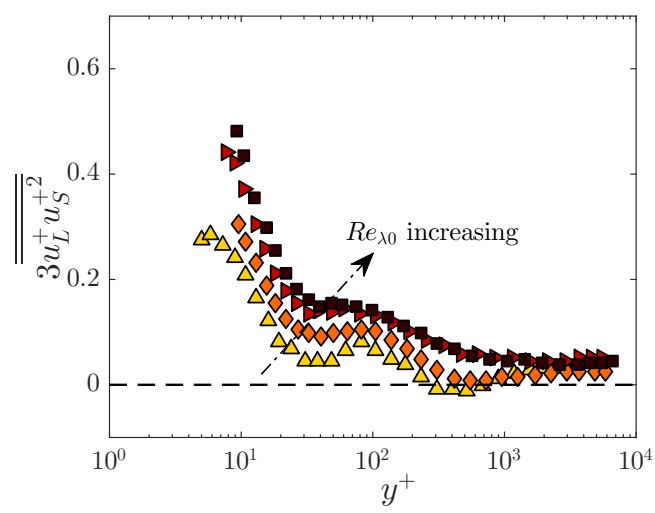

(d)

Figure 16: (Colour online) Variation of (a) the skewness factor, $S_{u}$, in comparison with the data from Mathis et al. (2009) and dominant terms of the scale-decomposed skewness (b) $\overline{\overline{u_{L}^{+3}}}$, (c) $\overline{\overline{u_{S}^{+3}}}$ and (d) $3 \overline{\overline{u_{L}^{+} u_{S}^{+2}}}$ across the boundary layer: $(\triangle)$ case $\mathrm{A} ;(\diamond)$ case B; $(\triangleright)$ case C; ( $\mathbf{a})$ case D. The inset for (b) shows a probability density function (pdf) of the large scales, $u_{L}$, for a representative case (case D) at $y^{+} \approx 100$ in comparison with a Gaussian distribution (blue smooth line).

layers without FST, it is shown by Metzger \& Klewicki (2001) that the local minima of the skewness in the near-wall region increases and becomes positive with increasing Reynolds number. Although the Reynolds numbers under consideration in the present study are not comparable to the highest value in their study, a similar trend in the local minima in the near-wall region is also observed for FST cases with increasing FST levels. This could be another supporting evidence for a possible analogy between the turbulent boundary layers under the FST effect and the high Reynolds number turbulent boundary layers without FST. The positive local minima observations here for the FST cases are similarly found in the results from Sharp et al. (2009).

The contributions from the large-scale term $\overline{\overline{u_{L}^{+3}}}$ (figure 16b) are almost always positive and seem to increase with FST levels. The positiveness of the large-scale contributions can be attributed to the fact that the distribution of large-scales in a grid-generated turbulence is slightly positively skewed (Mydlarski \& Warhaft 1996). This is also illustrated 
on the figure with the inset plot for the probability density function of large-scales for case $\mathrm{D}$ as a representative case and it is seen that the distribution is slightly positively skewed when compared to a Gaussian distribution. It is also worth to note that similar turbulence intensity cases (case A and case B or case C and case D) have similar largescale contributions. The substantial amount of contributions for all cases seems to occur at around the assumed start of the log-region and higher above, i.e. $y^{+}>30$. This region of dominance is expected to be due to the large scales in FST and their penetration and presence in the log-region of the boundary layer.

Figure 16c shows that the small-scale term $\overline{\overline{u_{S}^{+3}}}$ exhibits little variation across the majority of the boundary layer among different FST cases. The only place where there is any noticeable difference occurs around the near-wall region. The small-scale terms are actually found to locally contribute to the rise of the local minima of the skewness in the near-wall region (Mathis et al. 2011b). The figure shows that increasing the large-scale free-stream turbulence affects the small-scale contribution and this effect increases with increasing FST levels.

The final dominant term, $3 \overline{\overline{u_{L}^{+} u_{S}^{+2}}}$, shown in figure 16d, indicates that the amplitude modulation effect increases with increasing levels of FST. There also appears to be some collapse of the wall-normal location of the cross-term with increasing turbulence intensity. This suggests that there could be a finite limit to the levels of amplitude modulation that can be achieved. This trend with increasing FST levels is similar to the Reynolds number trend of a canonical boundary layer in Mathis et al. (2011b). This could imply that the present FST cases could be a limiting trend for a high Reynolds number flow where the turbulence intensity in the outer region is dominant. However, due to lack of available data, this is only proposed as a hypothesis.

Only for the two lower FST levels, the cross term can be seen to dip below zero in the outer region, however, it rises back above zero immediately after. This zero-crossing location has some implications on the phase relationship between large- and small-scales of the flow. Chung \& McKeon (2010) noted that the amplitude modulation coefficient, $R$, can be interpreted as a phase relationship between the large- and small-scales. Duvvuri \& McKeon (2015) showed that the phase relation naturally existing in the flow between the scales can be modified by introducing synthetic scales. They suggested that the envelope of all small-scales can be in-phase or out-of-phase with the synthetic large-scale depending on its location with respect to the location of the critical layer (this is the layer where the wave speed of the disturbance is equal to the local mean velocity).

Duvvuri \& McKeon (2015) found the zero-crossing location of their amplitude modulation coefficient to be around $y / \delta \approx 0.1$ (their $\delta$ definition is based on the location where $\left.U=0.99 U_{0}\right)$ which is very close to their critical layer location of $y / \delta \approx 0.07$. For the present cases, only the two lower turbulence intensity cases exhibit a zero crossing at around $y / \delta \approx 0.15$. This is further away from the wall compared to the single synthetic scale case. If we continue to interpret the zero-crossing as an indicator for the critical layer, then, the critical layer for these two FST cases is farther from the wall. This suggests that the critical layer tends to move towards the free-stream for higher intensity disturbances (and/or disturbances that are over a range of scales).

It can be seen that for higher FST cases, there is no zero-crossing in the amplitude modulation coefficient. This suggests that the small- and large-scales remain in-phase throughout the entire boundary layer when the forcing exceeds a certain amplitude. This implies that for broadband, high intensity forcing in the free-stream, it might not be possible to match the wave speed of a given disturbance to the mean velocity within the boundary layer and therefore the small- and large-scale remain in-phase throughout 
the entire layer. However, the wave speed of the disturbances in these FST cases is not known. Therefore, it is not possible to validate this observation at this time.

It was shown by Mathis et al. (2009) that single point measurements provide a reasonable estimate of the degree of amplitude modulation in the near-wall, with respect to a separate synchronised measurement at the outer peak, for a canonical turbulent boundary layer. However, the applicability of this to boundary layers forced by FST is yet to be established. A detailed study of the correlation between FST scales and the near-wall small scales requires simultaneous multi-point measurements, which is currently being pursued as a follow-up study.

\section{Conclusions}

The characteristics of a turbulent boundary layer under the effect of FST have been investigated. An active grid was used to generate the turbulence in the free-stream. In this study, FST cases were generated by rotating the grid rods at a specific motor scheme while running the wind tunnel at different inlet velocities. This resulted in four different FST cases that have similar length scales of $10 \delta$ but different turbulence intensity levels ranging from around $7 \%$ to around $13 \%$.

The effects of FST on the skin friction and boundary layer integral parameters are found to be consistent with the previous studies (Hancock \& Bradshaw 1983; Castro 1984; Stefes \& Fernholz 2004). Particular attention has been given to the scale interactions in the boundary layer in the presence of FST as an external disturbance. In order to do this, the present study has provided very near-wall boundary layer measurements with singlecomponent hot wire anemometry and this also promises insight for future investigations towards understanding the scale interactions in a turbulent boundary layer. By examining the energy spectra of the streamwise velocity fluctuations in the boundary layer, it has been inferred that the FST penetrates into the boundary layer and can go very near the wall. This has led to an overall increase in the streamwise velocity fluctuations in the boundary layer and the influence has been more prominent with increasing strength of the turbulence level in the free-stream. The near-wall peak amplitude has also kept increasing with the turbulence level. This has been found analogous to the trend in high Reynolds number canonical flows with increasing Reynolds number. Similar to these flows, this trend has also been attributed to the increasing influence of energetic large-scales in the outer region on the near-wall with increasing Reynolds number. We have also observed that the wall normal location of the near-wall broadband peak moves gradually outwards in wall units. Further studies and examinations are needed to validate this observation since it challenges the idea of having a fixed location $\left(y^{+} \approx 15\right)$ for the peak turbulence production in the near-wall region which has been accepted in turbulence studies to date.

The boundary layer forms a secondary peak in the logarithmic region under the effect of FST. This has been found similar to outer peak formation in high Reynolds number flows when there is enough scale separation. The strength of that peak has been observed to increase with increasing Reynolds number, directly related to the turbulence level in the free-stream for this particular study, and to move gradually outwards in wall units as also encountered in high Reynolds number canonical flows. All these similarities raise the potential for turbulent boundary layers under the effect of FST to represent high Reynolds number flows but at much lower Reynolds numbers which might help to overcome the limitations of the available experimental facilities.

The scales in the boundary layer have been decomposed using a cut-off wavelength filter as previously done by Hutchins \& Marusic (2007a) and Mathis et al. (2009). By investigating the scales separately, the increasing effect of large-scales on the near- 
wall region with increasing Reynolds number has been made clear. In addition to lowwavenumber energy (related to large-scales) superimposed in the near-wall region, there is also a modulating interaction between the scales which has been revealed by a skewness analysis. The cross-term of the scale-decomposed skewness factor, $3 \overline{\overline{u_{L}^{+} u_{S}^{+2}}}$, has been used as a tool to investigate modulation effects of large-scales on the envelope of the small-scale fluctuations. As the large-scales become more energetic with increasing Reynolds number, their modulation effect on the small-scales has been observed to increase. Introducing a wide range of large-scales into the flow, the phase relations between the scales are also expected to alter following the observations by Duvvuri \& McKeon (2015). Future efforts will be directed towards understanding the phase relations between the FST and the boundary layer scales as well as to further investigate modulating interactions between these scales.

We gratefully acknowledge the financial support from the European Research Council (ERC grant agreement no. 277472), EPSRC (grant ref no: EP/I037717/1), NSERC (postdoctoral fellowship for Ronald Hanson) and the Zonta International Foundation (Amelia Earhart fellowship for Eda Dogan). We are extremely grateful to Dr. Robert Bleischwitz for his major role in design and construction of the active grid. We also thank Luis Esteban Blay for carrying out Oil Film Interferometry measurements that validated the use of the Preston tube to estimate skin-friction. All data supporting this study are openly available from the University of Southampton repository at http://dx.doi.org/10.5258/SOTON/396902.

\section{Appendix A}

\section{REFERENCES}

BAlakumar, B.J \& AdRian, R.J 2007 Large- and very-large-scale motions in channel and boundary-layer flows. Philosophical Transactions of the Royal Society of London A: Mathematical, Physical and Engineering Sciences 365 (1852), 665-681.

Bandyopadhyay, Promode R. \& Hussain, A. K. M. F. 1984 The coupling between scales in shear flows. Physics of Fluids 27 (9), 2221-2228.

BLAIR, M. F. 1983 Influence of free-stream turbulence on turbulent boundary layer heat transfer and mean profile development, part i - experimental data. Journal of Heat Transfer 105, $33-40$.

Bruun, H H 1995 Hot-wire anemometry: principles and signal analysis. Oxford Univ. Press.

Brzek, B., Torres-Nieves, S., Lebrón, J., Cal, R., Meneveau, C. \& Castillo, L. 2009 Effects of free-stream turbulence on rough surface turbulent boundary layers. Journal of Fluid Mechanics 635, 207-243.

CAstro, I. P. 1984 Effects of free stream turbulence on low reynolds number boundary layers. Journal of Fluids Engineering 106, 298-306.

Chung, D. \& McKeon, B. J. 2010 Large-eddy simulation of large-scale structures in long channel flow. Journal of Fluid Mechanics 661, 341-364.

Coles, Donald 1956 The law of the wake in the turbulent boundary layer. Journal of Fluid Mechanics 1, 191-226.

Duvvuri, S. \& McKeon, B. J. 2015 Triadic scale interactions in a turbulent boundary layer. Journal of Fluid Mechanics 767, 4.

Fernholz, H.H. \& Finley, P.J. 1996 The incompressible zero-pressure-gradient turbulent boundary layer: An assessment of the data. Progress in Aerospace Sciences 32 (4), 245 311.

Ganapathisubramani, B., Hutchins, N., Monty, J. P., Chung, D. \& Marusic, I. 2012 


\begin{tabular}{|c|c|c|c|c|c|c|c|c|c|c|c|}
\hline FST Cases & $U_{0}(m / s)$ & $\sqrt{\overline{\overline{u_{0}^{2}}}} / U_{0}(\%)$ & $R e_{\lambda 0}$ & $L_{e} / \delta$ & $\delta(m)$ & $\beta$ & $H$ & $U_{\tau}(m / s)$ & $R e_{\tau}$ & $R e_{\theta}$ & $R e_{x}$ \\
\hline+ & 12.2 & 8.5 & 610 & 3.5 & 0.13 & 1.4 & 1.19 & 0.5 & 4300 & 5840 & $2.7 \times 10^{6}$ \\
\hline * & 8.1 & 7.7 & 390 & 4.7 & 0.13 & 1.0 & 1.22 & 0.33 & 2830 & 3840 & $1.8 \times 10^{6}$ \\
\hline$i$ & 10.2 & 8.2 & 510 & 2.7 & 0.15 & 1.6 & 1.19 & 0.42 & 4080 & 5120 & $2.3 \times 10^{6}$ \\
\hline$\sum_{3}^{3}$ & 12.2 & 8.5 & 610 & 2.5 & 0.15 & 1.7 & 1.18 & 0.5 & 4700 & 6000 & $2.7 \times 10^{6}$ \\
\hline$\diamond$ & 8.1 & 7.8 & 410 & 4.7 & 0.11 & 1.0 & 1.22 & 0.33 & 2480 & 3540 & $1.8 \times 10^{6}$ \\
\hline$\odot$ & 6.1 & 7.2 & 300 & 4.9 & 0.12 & 1.0 & 1.24 & 0.27 & 2120 & 2910 & $1.3 \times 10^{6}$ \\
\hline$\bullet$ & 8.2 & 7.9 & 410 & 4.0 & 0.13 & 1.1 & 1.22 & 0.34 & 2830 & 3770 & $1.8 \times 10^{6}$ \\
\hline$\oplus$ & 6.1 & 7.3 & 320 & 3.6 & 0.12 & 1.2 & 1.25 & 0.27 & 2045 & 2710 & $1.4 \times 10^{6}$ \\
\hline$\oplus$ & 9.8 & 12.4 & 600 & 0.7 & 0.13 & 4.4 & 1.19 & 0.41 & 3440 & 4330 & $2.2 \times 10^{6}$ \\
\hline$\boxplus$ & 6.0 & 11.1 & 400 & 0.6 & 0.15 & 4.1 & 1.19 & 0.27 & 2670 & 3400 & $1.3 \times 10^{6}$ \\
\hline$\otimes$ & 6.0 & 11.3 & 410 & 0.4 & 0.20 & 4.6 & 1.18 & 0.28 & 3550 & 3945 & $1.3 \times 10^{6}$ \\
\hline$\otimes$ & 11.6 & 13.0 & 750 & 0.5 & 0.18 & 5.0 & 1.16 & 0.49 & 5820 & 7470 & $2.6 \times 10^{6}$ \\
\hline$凶$ & 8.1 & 12.1 & 530 & 0.3 & 0.2 & 5.1 & 1.17 & 0.35 & 4645 & 5210 & $1.8 \times 10^{6}$ \\
\hline$\circledast$ & 6.0 & 11.3 & 400 & 0.3 & 0.17 & 4.8 & 1.18 & 0.27 & 3050 & 3855 & $1.3 \times 10^{6}$ \\
\hline 囷 & 8.0 & 11.9 & 510 & 0.5 & 0.18 & 4.4 & 1.17 & 0.34 & 4050 & 4920 & $1.8 \times 10^{6}$ \\
\hline (A) & 11.6 & 13.0 & 760 & 0.4 & 0.2 & 5.2 & 1.16 & 0.49 & 6450 & 7620 & $2.6 \times 10^{6}$ \\
\hline
\end{tabular}

Table 3: Free-stream and boundary layer parameters for FST cases apart from cases A-D. The measurements were performed at $43 M$ downstream of the active grid. Cases from $(+)$ to $(\oplus)$ were generated by using cut-out wings. Cases from $(\oplus)$ to $(\oplus)$ were generated by using solid-wings. $U_{0}$ : mean streamwise velocity of the free-stream, $\sqrt{\overline{u_{0}^{2}}} / U_{0}(\%)$ : turbulence intensity of the free-stream, $R e_{\lambda 0}$ : Reynolds number based on Taylor microscale, $L_{e} / \delta$ : turbulence length scale ratio defined by Hancock \& Bradshaw (1983), $\delta$ : boundary layer thickness, $\beta$ : FST parameter defined by Hancock \& Bradshaw $(1983), U_{\tau}$ : skin friction velocity, $R e_{\tau}$ : Reynolds number based on skin friction velocity, $R e_{\theta}$ : Reynolds number based on momentum thickness, $R e_{x}$ : Reynolds number based on streamwise distance, $x$. Detailed definitions of the parameters are given in $\S 3.1$ and $§ 3.2$.

Amplitude and frequency modulation in wall turbulence. Journal of Fluid Mechanics 712, 61-91.

Guala, M., Hommema, S. E. \& Adrian, R. J. 2006 Large-scale and very-large-scale motions in turbulent pipe flow. Journal of Fluid Mechanics 554, 521-542.

HANCOCK, P. E. \& Bradshaw, P. 1983 The effect of free-stream turbulence on turbulent boundary layers. Journal of Fluids Engineering 105(3), 284-289.

Hancock, P. E. \& Bradshaw, P. 1989 Turbulence structure of a boundary layer beneath a turbulent free stream. Journal of Fluid Mechanics 205, 45-76.

Hearst, R.J. \& Lavoie, P. 2015 The effect of active grid initial conditions on high reynolds number turbulence. Experiments in Fluids 56 (10), 1-20-.

Hearst, R. J., Buxton, O. R. H., Ganapathisubramani, B. \& Lavoie, P. 2012 Experimental estimation of fluctuating velocity and scalar gradients in turbulence. Experiments in Fluids 53 (4), 925-942. 
HoyAs, S. \& JimÉNEz, J. 2006 Scaling of velocity fluctuations in turbulent channels up to $\operatorname{Re}_{\tau}=2003$. Physics of Fluids 18.

Hutchins, Nicholas \& Marusic, Ivan 2007 a Large-scale influences in near-wall turbulence. Philosophical Transactions of the Royal Society A: Mathematical, Physical and Engineering Sciences 365 (1852), 647-664.

Hutchins, N., Nickels, T. B., Marusic, I. \& Chong, M. S. 2009 Hot-wire spatial resolution issues in wall-bounded turbulence. Journal of Fluid Mechanics 635, 103.

Hutchins, N., N. \& Marusic, I. $2007 b$ Evidence of very long meandering features in the logarithmic region of turbulent boundary layers. Journal of Fluid Mechanics 579, 1-28.

JACOBI, I. \& McKeon, B.J. 2013 Phase relationships between large and small scales in the turbulent boundary layer. Experiments in Fluids 54 (3), 1-13.

JAcoBi, I. \& McKeon, B. J. 2011a Dynamic roughness perturbation of a turbulent boundary layer. Journal of Fluid Mechanics 688, 258-296.

JACOBI, I. \& MCKeON, B. J. $2011 b$ New perspectives on the impulsive roughness-perturbation of a turbulent boundary layer. Journal of Fluid Mechanics 677, 179-203.

Jones, M. B., Marusic, I. \& Perry, A. E. 2001 Evolution and structure of sink-flow turbulent boundary layers. Journal of Fluid Mechanics 428, 1-27.

Kang, Hyung Suk, Chester, Stuart \& Meneveau, Charles 2003 Decaying turbulence in an active-grid-generated flow and comparisons with large-eddy simulation. Journal of Fluid Mechanics 480, 129-160.

Klewicki, Joe, Fife, Paul, Wei, Tie \& McMurtry, Pat 2007 A physical model of the turbulent boundary layer consonant with mean momentum balance structure. Philosophical Transactions of the Royal Society of London A: Mathematical, Physical and Engineering Sciences 365 (1852), 823-840.

KLEWICKI, JosePh C. 2010 Reynolds number dependence, scaling, and dynamics of turbulent boundary layers. Journal of Fluids Engineering 132 (9), 094001-094001.

Larssen, Jon V. \& Devenport, William J. 2011 On the generation of large-scale homogeneous turbulence. Experiments in Fluids 50, 1207-1223.

Ligrani, P. M. \& Bradshaw, P. 1987 Spatial resolution and measurement of turbulence in the viscous sublayer using subminiature hot-wire probes. Experiments in Fluids $\mathbf{5}$ (6), 407-417.

Makita, H. 1991 Realization of a large-scale turbulence field in a small wind tunnel. Fluid Dynamics Research 8(2) (1-4), 53.

Marusic, I., Mathis, R. \& Hutchins, N. 2010 Predictive model for wall-bounded turbulent flow. Science 329 (5988), 193-196.

Marusic, Ivan, Monty, Jason P., Hultmark, Marcus \& Smits, Alexander J. 2013 On the logarithmic region in wall turbulence. Journal of Fluid Mechanics $\mathbf{7 1 6 .}$

Mathis, R., Hutchins, N. \& Marusic, I. 2009 Large-scale amplitude modulation of the smallscale structures in turbulent boundary layers. Journal of Fluid Mechanics 628, 311-337.

Mathis, R., Hutchins, N. \& Marusic, I. 2011a A predictive inner-outer model for streamwise turbulence statistics in wall-bounded flows. Journal of Fluid Mechanics 681, 537-566.

Mathis, Romain, Marusic, Ivan, Hutchins, Nicholas \& Sreenivasan, K. R. $2011 b$ The relationship between the velocity skewness and the amplitude modulation of the small scale by the large scale in turbulent boundary layers. Physics of Fluids 23 (12).

Metzger, M. M. \& KLewicki, J. C. 2001 A comparative study of near-wall turbulence in high and low reynolds number boundary layers. Physics of Fluids 13 (3), 692-701.

Monkewitz, Peter A., Chauhan, Kapil A. \& Nagib, Hassan M. 2008 Comparison of mean flow similarity laws in zero pressure gradient turbulent boundary layers. Physics of Fluids $20(10),-$.

Mydlarski, L. \& WARHAFt, Z. 1996 On the onset of high-reynolds-number grid-generated wind tunnel turbulence. Journal of Fluid Mechanics 320, 331-368.

Nagib, Hassan M. \& Chauhan, Kapil A. 2008 Variations of von karman coefficient in canonical flows. Physics of Fluids 20 (10).

Patel, V. C. 1965 Calibration of the preston tube and limitations on its use in pressure gradients. Journal of Fluid Mechanics 23, 185-208.

Perry, A. E. \& Li, J. D. 1990 Experimental support for the attached-eddy hypothesis in zeropressure-gradient turbulent boundary layers. Journal of Fluid Mechanics 218, 405-438. 
Schlatter, Philipp \& ÖRLü, RAmis 2010 Quantifying the interaction between large and small scales in wall-bounded turbulent flows: A note of caution. Physics of Fluids 22 (5).

Sharp, N.S., Neuscamman, S. \& Warhaft, Z. 2009 Effects of large-scale free stream turbulence on a turbulent boundary layer. Physics of Fluids 21 (9).

Smits, Alexander J., McKeon, Beverley J. \& Marusic, Ivan 2011 High-reynolds number wall turbulence. Annual Review of Fluid Mechanics 43 (1), 353-375, arXiv: http://www.annualreviews.org/doi/pdf/10.1146/annurev-fluid-122109-160753.

Stefes, B. \& Fernholz, H.-H. 2004 Skin friction and turbulence measurements in a boundary layer with zero-pressure-gradient under the influence of high intensity freestream turbulence. European Journal of Mechanics - B/Fluids 23 (2), 303-318.

Thole, K. A. \& Bogard, D. G. 1996 High freestream turbulence effects on turbulent boundary layers. Journal of Fluids Engineering 118, 276-284.

Zagarola, M. V. \& Smits, Alexander J. 1998 Mean-flow scaling of turbulent pipe flow. Journal of Fluid Mechanics 373, 33-79. 